\title{
A matrix model for baryons and nuclear forces
}

\author{
Koji Hashimoto, ${ }^{a}$ Norihiro lizuka ${ }^{b}$ and Piljin $\mathbf{Y i}^{c}$ \\ ${ }^{a}$ Nishina Center, RIKEN, \\ Saitama 351-0198, Japan \\ ${ }^{b}$ Theory Division, CERN, \\ CH-1211 Geneva 23, Switzerland \\ ${ }^{c}$ School of Physics, Korea Institute for Advanced Study, \\ Seoul 130-722, Korea \\ E-mail: koji@riken.jp, norihiro.iizuka@cern.ch, piljin@kias.re.kr
}

ABSTRACT: We propose a new matrix model describing multi-baryon systems. We derive the action from open string theory on the wrapped baryon vertex D-branes embedded in the D4-D8 model of large $N_{c}$ holographic QCD. The positions of $k$ baryons are unified into $k \times k$ matrices, with spin/isospin of the baryons encoded in a set of $k$-vectors. Holographic baryons are known to be very small in the large 't Hooft coupling limit, and our model offers a better systematic approach to dynamics of such baryons at short distances. We compute energetics and spectra $(k=1)$, and also short-distance nuclear force $(k=2)$. In particular, we obtain a new size of the holographic baryon and find a precise form of the repulsive core of nucleons. This matrix model complements the instanton soliton picture of holographic baryons, whose small size turned out to be well below the natural length scale of the approximation involved there. Our results show that, nevertheless, the basic properties of holographic baryons obtained there are robust under stringy corrections within a few percents.

KEYWORDS: Gauge-gravity correspondence, QCD, 1/N Expansion

ARXiv EPRINT: 1003.4988 


\section{Contents}

1 Introduction 1

2 The matrix model 5

2.1 Action 5

$\begin{array}{lll}2.2 & \text { Derivation in gauge/gravity duality } & 6\end{array}$

$\begin{array}{lll}3 & \text { Single baryon } & 10\end{array}$

$\begin{array}{lll}3.1 \text { Hamiltonian } & 10\end{array}$

$\begin{array}{ll}3.2 \text { Single flavor } & 12\end{array}$

3.3 Two flavors 13

$\begin{array}{lll}3.4 & \text { Quantization } & 15\end{array}$

$\begin{array}{ll}\text { 3.5 Meson couplings } & 15\end{array}$

4 Two-body baryon interaction $\quad 17$

$\begin{array}{ll}4.1 \text { Two-baryon configuration } & 17\end{array}$

$\begin{array}{lll}\text { 4.1.1 Single flavor } & 17\end{array}$

$\begin{array}{ll}\text { 4.1.2 Two flavors } & 18\end{array}$

$\begin{array}{lll}4.2 & \text { Baryon interaction potential } & 18\end{array}$

$\begin{array}{lll}4.3 & \text { Universal repulsive core } & 20\end{array}$

5 Conclusion $\quad 23$

\section{Introduction}

Nuclear physics is one of the oldest branches of high energy physics, yet remains one of more difficult. Despite the fact that we know the underlying fundamental theory, i.e. QCD, we are still unable to predict, reliably and analytically, behavior of nuclei or even a single proton. The problem is of course that one must understand the strong-coupling regime of QCD, which by and large remains inaccessible except by large-scale lattice simulations. Traditionally, this set nuclear physics apart from the rest of high energy physics in many aspects. However, recent developments in the so-called gauge/gravity duality began to solve certain strongly coupled field theories, possibly including QCD or its close relatives, allowing the two communities merging with each other. In this paper, by using the gauge/gravity duality, we propose a new matrix model for the dynamics of multi-baryons, whereby we compute basic properties of holographic baryons, interaction with mesons, and ultimately nuclear forces.

What matrix? In our matrix model, the number of baryons is represented by the rank of the matrix. Therefore, for $k$-body baryons, it is a $\mathrm{U}(k)$ matrix model. If we pathintegrate out the off-diagonal elements of matrices, we are left with $k$ diagonal elements. 
It is these elements which represents the positions of the $k$ baryons. In addition, there are a pair of complex $k \times N_{f}$ rectangular matrices whose classical values are related to the size of baryon. Together, they form the well-known Atiyah-Drinfeld-Hitchin-Manin (ADHM) matrix of instantons.

Why matrix? In the large $N_{c}$ QCD, mesons are open strings and light degrees of freedom, with mass $\sim \mathcal{O}(1)$ [1], while baryons are solitons with large mass $\sim \mathcal{O}\left(N_{c}\right)[2]$. If we embed the large $N_{c}$ gauge theory into string theory, baryons are described by string theory solitons, i.e., D-branes [3, 4]. Therefore, the dynamics of multi-baryons are described by a multi-D-brane system, which is nothing but the $\mathrm{U}(k)$ matrix model. Our matrix model is along the line of the ADHM construction [5], which is the matrix description for multiinstantons in gauge theory, or equivalently along the line of D0-D4 quantum mechanics. ${ }^{1}$ In the context of QCD, one must of course deform the description appropriately. Before we explain these changes in detail, let us first briefly review the holographic QCD.

The application of gauge/gravity duality [8-10] to large $N_{c}$ QCD [1], i.e., holographic QCD was developed very much in recent years, especially thanks to the D4-D8 model by Sakai and Sugimoto [11]. This model starts with large $N_{c}$ number of D4-branes compactified on a thermal circle [12], representing pure QCD at the low energy, and incorporates $N_{f}$ species of massless quarks by intersecting $N_{f}$ pairs of D8- and anti-D8-branes [11]. In the large $N_{c}$ limit, the D4-branes are replaced by their dual geometry, and flavor D8 and anti-D8-branes are connected at the IR of the dual geometry, which is the geometrical realization of chiral symmetry breaking. The theory on the connected D8-anti-D8 brane in this dual geometry reproduces the low energy effective theory of light meson sector with remarkable accuracy.

This theory has only two independent input parameters, and are free of ambiguities which low energy chiral Lagrangians such as Skyrme model [13-15] possess. Furthermore, it contains not only the light vector meson but also infinite towers of massive vector meson, resulting in a model with infinite number of predictions. Most of these are irrelevant since at high energy the theory deviates from real QCD, but nevertheless there are many low energy processes which can be computed from this model. This aspect of the D4-D8 model $[11,16]$ is one of the most important points that sets the D4-D8 model aside from the other more generic AdS-inspired models.

Baryons in this D4-D8 setup turned out to be very interesting also. Baryons, in the large $N_{c}$ limit, are inherently nonperturbative objects with their mass scaling as $N_{c}$ [2] which is inverse of the genus expansion coupling constant. The holographic baryon in D4-D8 model is no exception, and can be introduced as instantonic soliton on the flavor D8-brane. Note that no new parameters are introduced in this step and all observables associated with baryons are computable by the D4-D8 model. ${ }^{2}$

Following the original idea [3, 4], we recall that baryons can be also thought of as

\footnotetext{
${ }^{1}$ The relevance of such a matrix model for baryons was previously emphasized by one of the authors $[6,7]$.

${ }^{2}$ Employing this picture, static properties [17, 18], interactions with mesons [17, 19], electromagnetic form factors [20, 21], and more recently nucleon-nucleon potential [22, 23] have been derived again with remarkable accuracy. Although most literatures considered nucleons, higher isospin baryons can also be treated on equal footing $[24,25]$.
} 
D4-branes wrapped on the compact $S^{4}$ that surrounds the QCD $N_{c}$ D4-branes. Let us call them D4'-branes to distinguish them from those responsible for QCD. As such, its effective dynamics in the large $N_{c}$ limit involve open strings with both ends on the D4'-branes and also those connecting the D4'-branes and the D8-branes. The dynamics of $k$ baryons would be $\mathrm{U}(k)$ gauge theory, and if we consider only the zero mode ${ }^{3}$ along the $S^{4}$ on which the D4' and the D8 are wrapped on, the theory on the D4'-branes reduces to a $0+1$ dimensional matrix model. Motivated by this viewpoint, in this paper, we propose a new $\mathrm{U}(k)$ matrix model for holographic baryons.

To illustrate our matrix model, let us remember a simple D-brane bound state of the $\mathrm{D} p-\mathrm{D}(p+4)$ system and that we have two descriptions for this system. One is the solitonic description for the $\mathrm{D} p$-branes as instanton solitons in the $\mathrm{D}(p+4)$-brane gauge theory. This description is natural when gauge fields are weakly varying, namely at long distance scale with $\rho \gg l_{s}$ where $\rho$ is the size of the instanton soliton and $l_{s}$ is the string length. The other is the open string matrix theory viewpoint, whereby the ADHM construction of the instanton soliton is naturally derived [27, 28]. This approach is more natural at a short distance scale, $\rho \ll l_{s}$. For fully supersymmetric case, the two descriptions are equivalent for many purposes.

Our matrix model for the $k$ D4'-branes follows the latter viewpoint and is a D4'D8 matrix theory compactified on a common $S^{4}$. There are several differences between our matrix model for baryons and the usual $\mathrm{D} p$ - $\mathrm{D}(p+4)$-brane system. First, in our matrix model, there is a Chern-Simons (CS) term (supersymmetric versions were studied in $[29,30])$. This term originates from the fact that there is a Ramond-Ramond (RR) flux on the $S^{4}$ on which the D4'-branes are wrapped, and is in fact the same type of term that allowed Witten to identify wrapped D5-branes as baryon vertices in AdS/CFT description of maximally supersymmetric Yang-Mills theory [3]. For us, this term turns out to play a crucial role in dynamics of baryons in general and in the baryon-baryon interactions in particular. Second, since the D4'-branes are living on the D8-brane at the IR bottom of the warped geometry, we need to take into account the warped geometry to derive the $\mathrm{U}(k)$ matrix model. The point is that if we put the $k$ baryons at short distances, the warp factor approaches almost constant values, therefore the effects of the warped geometry is simply just rescaling of a coupling constant plus mass terms. This in turns implies that the supersymmetry is broken explicitly by the dual geometry $[12,31]$ of the QCD D4-branes. In practice, the supersymmetry breaking would manifest as various mass terms and potential energy in the would-be vacuum moduli space.

One motivation for us is that such a matrix formulation can easily accommodate a large number of baryons. One of more grey area of nuclear physics, from the purely theoretical viewpoint, is how one handles many-nucleon systems such as nuclei. The fundamental theory of QCD is even less effective there. The matrix model of ours can be written down immediately for all $k$, and should contain in principle, not only 2-body interactions

\footnotetext{
${ }^{3}$ The subtlety of taking of a decoupling limit of the non-zero modes along $S^{4}$ is a long-standing problem in holographic QCD. As a result, it is obscure why in low energy effective theories such heavy objects like baryons are well-described. For one possible explanation concerning supersymmetries offered by one of the authors, see [26].
} 
but arbitrary $k$-body interactions built-in from the beginning. This may open up a novel window for dealing with finite $k$ nuclei and large $k$ physics such as necessary for neutron star, in term of strongly-coupled QCD.

Another motivation, which is perhaps more practical for now, is already apparent in the holographic soliton picture of baryon $[17,18]$. Remember that the holographic size of the solitonic baryon is $\simeq 9.6 /\left(M_{\mathrm{KK}} \sqrt{\lambda}\right)$, where $\lambda$ is the 't Hooft coupling and $M_{\mathrm{KK}} \sim 1 \mathrm{GeV}$ is the mass scale of the vector mesons $[17,18]$. Although this size is larger than the Compton size of the baryon $\sim 1 /\left(M_{\mathrm{KK}} N_{c} \lambda\right)$, obeying the usual criterion for validity of field theory soliton picture, it is unfortunately as small as the local string scale $l_{s}^{\text {eff }} \sim 1 /\left(M_{\mathrm{KK}} \sqrt{\lambda}\right)$. One should be dubious whether the field theory soliton is really accurate enough for such a small object. The supersymmetry breaking scale is $\sim 1 / M_{\mathrm{KK}}$, so the supersymmetry is approximately valid at the very short distance such as the string scale, so one hope for relative stability of the baryon physics in interpolating between the string scale and the QCD meson scale [26]. Nevertheless, we cannot expect quantitative agreement for all observables.

Open string description of D-brane interactions is deemed to be relevant for distance scale below string scale, so we have a motivation and an opportunity here to reexamine holographic baryons from a D4'-D8 (compactified on $S^{4}$ ) matrix description. With supersymmetry broken at $M_{K K}$ and below, one should in general expect the solitonic viewpoint and the matrix viewpoint would disagree quantitatively; it would be interesting to compare our matrix description with the previous soliton description.

In section 2, we drive the matrix model by starting with D4'-D8 gauge theory compactified on $S^{4}$, and extracting leading order supersymmetry-breaking effects at very short distance. The action we find is valid at distance $l_{s}^{\text {eff }}$ and below. To show the effectiveness of our matrix model, we display only two simple examples in this paper: energy functions of static configurations for $k=1$ and $k=2$, for one and two flavor(s).

In section 3 , we discuss $k=1$ and determine the holographic "size" of the baryon, value of which affects numerous observable quantities. This parallels the energy function estimates for the solitonic baryon in spirit but differs in detail. For two or more flavors, we find the energy function with a different numerical coefficient, leading to a new estimate for the holographic size which is larger by a factor of $(5 / 4)^{1 / 4}$ than the soliton result. We discuss its implications. With a singe flavor, for which the soliton model has no computation due to lack of finite and smooth self-dual U(1) instanton solutions, we find a smaller but still non-vanishing size.

In section 4 , where $k=2$ is studied, we compute a baryon-baryon potential at short distance for two flavors. Interestingly, integrating out the auxiliary gauge potential in $0+1$ dimension turns out to give a universal repulsive core of the nuclear force. The core consists of three terms; the isospin-independent central term, isospin-dependent central term, and isospin-dependent tensor term. The computation here is far simpler than the soliton computation, yet gives the same type of results, except that numerical coefficient of the latter two turn out to be larger by a factor of $5 / 4$. We explain this in simple terms based on the above $k=1$ results. We also show that the repulsive core is universal for any baryon state for the two-flavor case.

In section 5, we close with discussions on possible ramifications of this new model of baryons and baryon dynamics. 


\section{The matrix model}

We derive the action of the matrix quantum mechanics in this section, which will be used for getting the baryon spectrum (section 3), the nucleon-nucleon potential and the universal repulsive core (section 4 ).

The matrix model is nothing but the low energy effective action on the $k$ D4'-branes embedded in flavor $N_{f}$ D8-branes in Witten's geometry. Those who are not interested in the string-theoretical derivation are advised to see only the action (in section 2.1), skip the rest and go directly to section 3 .

\subsection{Action}

The matrix model action we derive is a $\mathrm{U}(k)$ quantum mechanics,

$$
\begin{aligned}
S= & \frac{\lambda N_{c} M_{\mathrm{KK}}}{54 \pi} \int d t \operatorname{tr}_{k}\left[\left(D_{0} X^{M}\right)^{2}-\frac{2}{3} M_{\mathrm{KK}}^{2}\left(X^{4}\right)^{2}+D_{0} \bar{w}_{i}^{\dot{\alpha}} D_{0} w_{\dot{\alpha} i}-\frac{1}{6} M_{\mathrm{KK}}^{2} \bar{w}_{i}^{\dot{\alpha}} w_{\dot{\alpha} i}\right. \\
& \left.+\frac{3^{6} \pi^{2}}{4 \lambda^{2} M_{\mathrm{KK}}^{4}}(\vec{D})^{2}+\vec{D} \cdot \vec{\tau}_{\dot{\beta}}^{\dot{\alpha}} \bar{X}^{\dot{\beta} \alpha} X_{\alpha \dot{\alpha}}+\vec{D} \cdot \vec{\tau}_{\dot{\beta}}^{\dot{\alpha}} \bar{w}_{i}^{\dot{\beta}} w_{\dot{\alpha} i}\right] \\
& +N_{c} \int d t \operatorname{tr}_{k} A_{0} .
\end{aligned}
$$

Here $\lambda=N_{c} g_{\mathrm{QCD}}^{2}$ is the 'tHooft coupling constant, and $M_{\mathrm{KK}}$ is the unique dimension-ful constant. The dynamical fields are $X^{M}$ and $w$, while $\vec{D}$ and $A_{0}$ are auxiliary fields. All the fields are bosonic. We claim that this matrix model describes the $k$-baryon system, according to the holographic principle in string theory.

Our 1-dimensional matrix model is a deformed ADHM matrix model. The ADHM matrix model has been extensively studied in the context of D-branes in string theory and instanton calculus (for a concise review, see [32]). Our theory is deformed in the following two points:

- Addition of the CS coupling. The last term of the action (2.1) is a CS term in 1 dimension.

- Mass deformation. A part of the dynamical fields, $X^{4}$ and $w$, are massive, in contrast to the standard ADHM matrix model.

We will describe how the ADHM matrix model and these deformations appear in the holographic QCD, together with the detailed derivation of the coefficients in the action. Note that in the absence of the field $w$, the CS term and the mass term, our matrix model looks close to the BFSS Matrix theory for M-theory [33] or the IKKT matrix model [34], as the integration of the auxiliary field $D$ results in a potential of a commutator type, $\operatorname{tr}\left([X, X]^{2}\right)$.

The symmetry of this matrix quantum mechanics is

$$
\mathrm{U}(k) \times \mathrm{SU}\left(N_{f}\right) \times \mathrm{SO}(3)
$$

where the first $\mathrm{U}(k)$ is a local symmetry with which the gauge field $A_{0}$ is associated, and the remaining $\mathrm{SU}\left(N_{f}\right) \times \mathrm{SO}(3)$ is a global symmetry. $k$ refers to the number of baryons of 


\begin{tabular}{|c|c|c|c|c|}
\hline field & index & $\mathrm{U}(k)$ & $\mathrm{SU}\left(N_{f}\right)$ & $\mathrm{SU}(2) \times \mathrm{SU}(2)$ \\
\hline$X^{M}(t)$ & $M=1,2,3,4$ & $\mathrm{adj}$. & $\mathbf{1}$ & $(\mathbf{2}, \mathbf{2})$ \\
\hline$w_{\dot{\alpha} i}(t)$ & $\dot{\alpha}=1,2 ; i=1, \cdots, N_{f}$ & $\mathbf{k}$ & $\mathbf{N}_{\mathbf{f}}$ & $(\mathbf{1}, \mathbf{2})$ \\
\hline$A_{0}(t)$ & & $\mathrm{adj}$. & $\mathbf{1}$ & $(\mathbf{1}, \mathbf{1})$ \\
\hline$D_{s}(t)$ & $s=1,2,3$ & $\mathrm{adj}$. & $\mathbf{1}$ & $(\mathbf{1}, \mathbf{3})$ \\
\hline
\end{tabular}

Table 1. Fields in the matrix model.

the system, $N_{f}$ is the number of flavors in QCD, and $\mathrm{SO}(3)$ is the rotational symmetry of our space in which the baryons live. The notation of the action is better-understood if we embed the rotational symmetry as

$$
\mathrm{SO}(3) \subset \mathrm{SO}(4) \sim \mathrm{SU}(2) \times \mathrm{SU}(2)
$$

where the additional dimension in fact corresponds to the holographic dimension. This $\mathrm{SO}(4)$ is broken down to the $\mathrm{SO}(3)$ by the mass deformation.

We summarize the representation of the fields in the table 1 . The indices with respect to the $\mathrm{U}(k)$ gauge group are implicit. In the action, the trace is over these $\mathrm{U}(k)$ indices. One can think of the flavor symmetry as $\mathrm{U}\left(N_{f}\right)$, while the overall $\mathrm{U}(1)$ part of it is identical to the overall $\mathrm{U}(1)$ part of the gauge symmetry $\mathrm{U}(k)$, as seen in how they act on the bifundamental field $w$.

In the action, the definition of the covariant derivatives is $D_{0} X^{M} \equiv \partial_{0} X^{M}-i\left[A_{0}, X^{M}\right]$, $D_{0} w \equiv \partial_{0} w-i w A_{0}, D_{0} \bar{w} \equiv \partial_{0} \bar{w}+i A_{0} \bar{w}$, and $\tau^{s}(s=1,2,3)$ is the Pauli matrix. The spinor indices of $X$ are defined as $X_{\alpha \dot{\alpha}} \equiv X^{M}\left(\sigma_{M}\right)_{\alpha \dot{\alpha}}$ and $\bar{X}^{\dot{\alpha} \alpha} \equiv X^{M}\left(\bar{\sigma}_{M}\right)^{\dot{\alpha} \alpha}$ where $\sigma_{M}=(i \vec{\tau}, 1)$ and $\bar{\sigma}_{M}=(-i \vec{\tau}, 1)$. We follow the notation of [32].

\subsection{Derivation in gauge/gravity duality}

Our matrix model (2.1) is nothing but a low energy effective field theory on D-branes. The D-branes of our concern are D4'-branes wrapping $S^{4}$ of a background geometry given by Witten [12] (the metric originally given in [31]). This D4'-brane is called "baryon vertex" $[3,4]$ in the gravity side of the AdS/CFT duality, which corresponds, as the name shows, to a baryon in the field theory side. As we are dealing with a $k$-baryon system, we place these $k$ D4'-branes close to each other. We are going to derive the effective action of this collection of the D4'-branes wrapping the $S^{4}$, via a standard technique in string theory.

The D-brane action is affected not only by the geometry and the background flux, but also by the presence of the probe $N_{f}$ D8-branes which are responsible for quarks a la Sakai and Sugimoto [11]. We have additional strings connecting the baryon D4'-brane and the flavor D8-brane. From the viewpoint of the baryon D4'-brane effective field theory, this string provides the field $w$ in the bi-fundamental representation. Together with the field $X^{M}$ in the adjoint representation whose diagonal eigenvalues specify the location of the D4'-branes in the transverse directions (but longitudinal to the D8-brane worldvolume) and thus the location of the baryons in our real space, the matrix model action is written. 
Since the D4'-branes are completely inside the worldvolume of the flavor D8-branes, the matrix model is very close to the so-called supersymmetric ADHM matrix model which is nothing but the effective action of $k$ D-instantons on $N_{f}$ D3-branes in flat spacetime. The deformation is due to the curved geometry and the flux, which break the supersymmetry explicitly. Here we keep only the bosonic fields, which is enough for computing classical quantities.

Although the D4'-branes (and the D8-branes) wrap the $S^{4}$, we trivially reduce the $S^{4}$ dependence (a dimensional reduction with the assumption of no dependence along $S^{4}$ ), so that the resulting action is in one dimension, i.e. only time direction.

The background geometry and the flux given by Witten [12] (and Gibbons and Maeda [31]) are written as

$$
\begin{aligned}
& d s^{2}=(U / R)^{3 / 2}\left(\eta_{\mu \nu} d x^{\mu} d x^{\nu}+f(U) d \tau^{2}\right)+(R / U)^{3 / 2}\left(f(U)^{-1} d U^{2}+U^{2} d \Omega_{4}^{2}\right), \\
& e^{\phi}=g_{s}(U / R)^{3 / 4}, \quad F_{4}=d C_{3}=\frac{2 \pi N_{c}}{V_{4}} \epsilon_{4},
\end{aligned}
$$

where $f(U) \equiv 1-U_{\mathrm{KK}}^{3} / U^{3}$, and $R^{3} \equiv \pi g_{s} N_{c} l_{s}^{3} . V_{4} \equiv 8 \pi^{2} / 3$ is the volume of the $S^{4}$, and $\epsilon_{4}$ is the volume form on it. ${ }^{4}$ The $\tau$ direction is compactified with the period $\tau \sim \tau+2 \pi / M_{\mathrm{KK}}$ where $M_{\mathrm{KK}} \equiv(3 / 2) U_{\mathrm{KK}}^{1 / 2} R^{-3 / 2}$, so that the geometry is everywhere smooth. The relations to the QCD variables are

$$
R^{3}=\frac{1}{2} \frac{g_{\mathrm{YM}}^{2} N_{c}}{M_{\mathrm{KK}}} l_{s}^{2}, \quad U_{\mathrm{KK}}=\frac{2}{9} g_{\mathrm{YM}}^{2} N_{c} M_{\mathrm{KK}} l_{s}^{2}, \quad g_{s}=\frac{1}{2 \pi} \frac{g_{\mathrm{YM}}^{2}}{M_{\mathrm{KK}}} \frac{1}{l_{s}}
$$

Convenient coordinates used in $[11,16]$ are

$$
U^{3}=U_{\mathrm{KK}}^{3}+U_{\mathrm{KK}} r^{2}, \quad \theta \equiv \frac{3}{2} \frac{U_{\mathrm{KK}}^{1 / 2}}{R^{3 / 2}} \tau, \quad y+i z \equiv r e^{i \theta} .
$$

The flavor D8-branes are located at $y=0$.

Below we derive the action, by looking at, first, the background RR flux, and second, the effect of the background geometry.

Chern-Simons term. The important term is the last term of the matrix model (2.1), which is a CS term in $0+1$ dimension. Using the background RR flux $(2.5)^{5}$ we can compute the CS term on the D4'-brane as

$$
\begin{aligned}
S & =\frac{1}{2 \pi} \frac{1}{2 \cdot 3 !} \int d^{5} \xi \operatorname{tr} \epsilon^{\mu_{1} \mu_{2} \mu_{3} \alpha \beta} C_{\mu_{1} \mu_{2} \mu_{3}} F_{\alpha \beta}=\frac{1}{2 \pi} \int d t \operatorname{tr} A_{0} \int F_{4} \\
& =N_{c} \int d t \operatorname{tr} A_{0} .
\end{aligned}
$$

\footnotetext{
${ }^{4}$ We use the standard normalization for the forms, $C_{3}=(1 / 3 !) \quad C_{i j k} d x^{i} \wedge d x^{j} \wedge d x^{k}$ and $F_{4}=$ $(1 / 3 !) \partial_{\alpha} C_{i j k} d x^{\alpha} \wedge d x^{i} \wedge d x^{j} \wedge d x^{k}$.

${ }^{5}$ We are using the normalization of the RR field in which the RR charge is measured in units of $2 \pi$, see appendix A of [11].
} 
Note that the overall factor $N_{c}$ shows that the D4'-brane should be supplied with $N_{c}$ fundamental strings (the end point of the D4'-D8 string serves as an electric charge on the D4'-brane), which means that the D4'-brane is indeed a baryon $[3,4] .{ }^{6}$

Interestingly, this 0+1-dimensional CS term was used in [29] and [30] for the ADHM matrix model for supersymmetric dyonic instantons with a CS term in 5 dimensions. There, the CS term was argued for heuristically ([29] studied fermions and anomalies to reach the 0+1-dimensional CS term). Here we have derived the 0+1-dimensional CS term from string theory.

Mass terms and overall normalization. The Dirac-Born-Infeld part of the action for a single D4'-brane is

$$
S=-T_{\mathrm{D} 4} \int d^{5} \xi e^{-\phi} \sqrt{-\operatorname{det}\left(G_{M N}+2 \pi \alpha^{\prime} F_{M N}\right)}
$$

We consider a D4'-brane situated at $y=0$ which wraps the $S^{4}$. Then

$$
S=-\frac{T_{\mathrm{D} 4}}{g_{s}} \int d t V_{4}\left((R / U)^{3 / 2} U^{2}\right)^{2}(U / R)^{-3 / 4} \sqrt{-G_{00}}
$$

with $V_{4} \equiv 8 \pi^{2} / 3$ the volume of a unit four-sphere, where the induced metric is

$$
G_{00}=-\left(\frac{U}{R}\right)^{3 / 2}\left(1-\left(\partial_{0} X^{i}\right)^{2}\right)+\frac{4}{9}\left(\frac{R}{U}\right)^{3 / 2} \frac{U_{\mathrm{KK}}}{U}\left(\partial_{0} Z\right)^{2} .
$$

The index of $X^{i}$ runs for our 3 -dimensional space, $i=1,2,3$. So, we obtain

$$
S=-\frac{T_{\mathrm{D} 4}}{g_{s}} \int d t \frac{8 \pi^{2}}{3} R^{3} U \sqrt{1-\left(\partial_{0} X^{i}\right)^{2}-\frac{4}{9} \frac{R^{3} U_{\mathrm{KK}}}{U^{4}}\left(\partial_{0} Z\right)^{2}} .
$$

with $T_{D p}=2 \pi /\left(2 \pi \sqrt{\alpha^{\prime}}\right)^{p+1}$.

We expand this for small $Z$ and small $X$. Using the expansion $U=U_{\mathrm{KK}}(1+$ $\left.(1 / 3) U_{\mathrm{KK}}^{-2} Z^{2}+\mathcal{O}\left(Z^{4}\right)\right)$, and a redefinition

$$
X^{4} \equiv \frac{2}{3}\left(\frac{R}{U_{\mathrm{KK}}}\right)^{3 / 2} Z
$$

we obtain a quadratic Lagrangian

$$
S=\frac{\lambda N_{c} M_{\mathrm{KK}}}{27 \pi} \int d t\left[-1+\frac{1}{2}\left(\partial_{0} X^{i}\right)^{2}+\frac{1}{2}\left(\partial_{0} X^{4}\right)^{2}-\frac{1}{3} M_{\mathrm{KK}}^{2}\left(X^{4}\right)^{2}\right]
$$

${ }^{6}$ This Chern-Simons term is also important in producing the correct statistics of baryons, which should be either fermionic or bosonic depending on whether $N_{c}$ is odd or even. For detail of nucleon statistics in our matrix model, see ref. [35]. 
In this way, the overall normalization of the matrix model action, ${ }^{7}$ as well as the mass term for the field $X^{4}$, are provided in the matrix model (2.1). This mass term originates entirely from the expansion of $U$ sitting in front of the square-root in (2.12).

This leaves the mass term for $w$. We assume that it is

$$
-\frac{\lambda N_{c} M_{\mathrm{KK}}}{54 \pi} \int d t \frac{1}{6} M_{\mathrm{KK}}^{2} \bar{w}_{i}^{\dot{\alpha}} w_{\dot{\alpha} i}
$$

to be added to (2.14), which completes the quadratic part of the matrix action in (2.1). This (2.16) is an educated guess via a comparison with the soliton picture (though in principle one can derive this by computing string scattering amplitudes). In fact, as we will see in the next section, the $X^{4}$ mass term in (2.14) coincides with the soliton picture, and the following argument suggests that $w$ mass also coincides.

Recall that $\left(X^{i}, X^{4}, w\right)$ form the ADHM data for instantons. When the instanton size is very small compared to the supersymmetry-breaking scale $1 / M_{K K}$, the background geometry is effectively flat with approximate supersymmetry. The D4'-D8 system will inherit supersymmetry, and thus the data $\left(X^{i}, X^{4}, w\right)$ is equivalent to the supersymmetric instanton on the D8-branes; At this zero-th order, the instanton picture of the D4'-brane on the D8-branes is still valid. Note that this is not yet the baryon but merely a purely magnetic instanton solution. Then, we turn to the effect of supersymmetry breaking at $M_{K K}$ scale, and evaluate the potential energy of the instanton at the first order. This is in fact one way of obtaining the mass term for $X^{4}$ in (2.1). Similarly, using the fact that $|w|^{2} / 2$ is the size squared for a single instanton, we find (2.16) for the matrix model. ${ }^{8}$

Commutator term. Finally, let us compute the coefficient in front of $\vec{D}^{2}$ which is related to the coefficient in front of the famous commutator term $[X, X]^{2}$ in D-brane quantum mechanics.

Let us expand the generic $\mathrm{D} p$-brane action (2.9) to the quadratic order,

$$
S=-T_{\mathrm{D} p} \int d^{p+1} \xi e^{-\phi} \sqrt{-\operatorname{det} G_{M N}} \frac{1}{4}\left(2 \pi \alpha^{\prime}\right)^{2} F_{M N} F_{P Q} G^{M P} G^{N Q} .
$$

We now make a dimensional reduction to get the commutator term from the YM kinetic action. The relevant formula for the dimensional reduction is $2 \pi \alpha^{\prime} A_{M}=X^{N} G_{M N}$ for

\footnotetext{
${ }^{7}$ Note that higher order terms in $X^{4}$ are not suppressed by $1 / \lambda$. In fact, the leading correction to the mass term is

$$
+\frac{1}{3} M_{\mathrm{KK}}^{2}\left(X^{4}\right)^{2}-\frac{1}{9} M_{\mathrm{KK}}^{4}\left(X^{4}\right)^{4}+\cdots
$$

In the following, we assume that the magnitude of $X^{4}$ is small so that we can ignore the higher corrections. In fact, as we consider the wave function of this $X^{4}$ after the quantization around the vacuum of the matrix model, we obtain a Gaussian wave function with the width suppressed by $1 / N_{c}$ which is quite small, and this approximation is valid.

${ }^{8}$ One may recall that the soliton representation of the holographic baryon has a Coulombic potential as well $[17,18]$. This is not apparent yet in the matrix model but will arise by integrating out the gauge potential $A_{0}$ of $\mathrm{D} 4$ '. The crucial difference from the mass terms is that the Coulombic energy arises from a quadrature of an electric field (in the soliton picture) which is itself a first order deviation. We will see later that, perhaps because of this, the Coulombic energy differs quantitatively in the two pictures.
} 
diagonal metrics. Then, the action is

$$
\begin{aligned}
L \propto & 2 G^{00} G_{i j} D_{0} X^{i} D_{0} X^{j}+2 G^{00} G_{z z} D_{0} Z D_{0} Z \\
& -\left[X^{i}, X^{j}\right]\left[X^{k}, X^{l}\right] G_{i k} G_{j l} \frac{1}{\left(2 \pi \alpha^{\prime}\right)^{2}}-2\left[X^{i}, Z\right]\left[X^{j}, Z\right] G_{i j} G_{z z} \frac{1}{\left(2 \pi \alpha^{\prime}\right)^{2}} \\
\propto & \left(D_{0} X^{i}\right)^{2}+\left(D_{0} X^{4}\right)^{2}+\frac{1}{2} \frac{1}{\left(2 \pi \alpha^{\prime}\right)^{2}}\left(\left[X^{i}, X^{j}\right]^{2}+2\left[X^{i}, X^{4}\right]^{2}\right)\left(\frac{U_{\mathrm{KK}}}{R}\right)^{3} \\
= & \left(D_{0} X^{M}\right)^{2}+\frac{2}{3^{6} \pi^{2}} \lambda^{2} M_{\mathrm{KK}}^{4}\left[X^{M}, X^{N}\right]^{2} .
\end{aligned}
$$

On the other hand, the commutator term can be written by using the auxiliary field $\vec{D}$. If we start from the action of $[32]^{9}$

$$
S=c \int d t \operatorname{tr}\left[2\left(2 \pi \alpha^{\prime}\right)^{2}(\vec{D})^{2}+\vec{D} \cdot \vec{\tau} \dot{\alpha^{\prime}} \overrightarrow{a^{\prime}} \dot{\beta} \alpha a_{\alpha \dot{\alpha}}^{\prime}\right]
$$

then by integrating out the field $\vec{D}$ we obtain

$$
S=c \int d t \operatorname{tr}\left[\frac{1}{16 \pi^{2} \alpha^{\prime 2}}\left[a_{m}^{\prime}, a_{n}^{\prime}\right]^{2}\right]
$$

Comparing this with the normalization we obtained in (2.18), we obtain the expression for the commutator term of our matrix model as

$$
\frac{\lambda N_{c} M_{\mathrm{KK}}}{54 \pi} \int d t \operatorname{tr}_{k}\left[\frac{3^{6} \pi^{2}}{4 \lambda^{2} M_{\mathrm{KK}}^{4}}(\vec{D})^{2}+\vec{D} \cdot \vec{\tau}_{\dot{\beta}}^{\dot{\alpha}} \bar{X}^{\dot{\beta} \alpha} X_{\alpha \dot{\alpha}}\right]
$$

The $w$ term coupled to $\vec{D}$ is written down in [32] and we can just use it with the same normalization as the $\vec{D} X X$ term.

\section{Single baryon}

In this section, we study the $k=1$ case, i.e. a single baryon. Our quantum mechanics directly gives a spectrum of the baryon.

First, we evaluate the Hamiltonian of the quantum mechanics with $k=1$. Then we analyze the vacuum of the system for $N_{f}=1$ and $N_{f}=2$ respectively. For $N_{f}=2$ system, we calculate the baryon spectrum. The computation of the spectrum, as well as the quantization procedure, closely follow the soliton approach of [17, 18], although the derivation of the Hamiltonian is different. Finally we discuss meson couplings.

\subsection{Hamiltonian}

Let us compute the Hamiltonian for a single baryon $k=1$, with generic $N_{f}$.

First we explicitly integrate out auxiliary fields $\vec{D}$ and $A_{0}$. As for the terms including the field $\vec{D}$, since for $k=1$ the field $X$ is now not a matrix but a number, all the $X$

\footnotetext{
${ }^{9}$ In [32] the field $\vec{D}$ is anti-Hermitian, while our $\vec{D}$ is defined to be Hermitian.
} 
couplings drop off, and we obtain

$$
\begin{aligned}
S_{\vec{D}}=\frac{\lambda N_{c} M_{\mathrm{KK}}}{54 \pi} \int d t \frac{-\lambda^{2} M_{\mathrm{KK}}^{4}}{3^{6} \pi^{2}} & {\left[\sum\left(\vec{\tau}_{\dot{\beta}}^{\dot{\alpha}} \bar{w}_{i}^{\dot{\beta}} w_{\dot{\alpha} i}\right)^{2}\right] } \\
=\frac{\lambda N_{c} M_{\mathrm{KK}}}{54 \pi} \int d t \frac{-\lambda^{2} M_{\mathrm{KK}}^{4}}{3^{6} \pi^{2}}[ & 4 w_{1}^{i}\left(w_{2}^{i}\right)^{*} w_{2}^{j}\left(w_{1}^{j}\right)^{*} \\
& \left.+\left(w_{1}^{i}\left(w_{1}^{i}\right)^{*}\right)^{2}+\left(w_{2}^{i}\left(w_{2}^{i}\right)^{*}\right)^{2}-2 w_{1}^{i}\left(w_{1}^{i}\right)^{*} w_{2}^{j}\left(w_{2}^{j}\right)^{*}\right],
\end{aligned}
$$

where, the first sum is over three Pauli matrices and we omit the dots in the dotted spinor, $\dot{\alpha}=1,2 . S_{\vec{D}}$ gives a so-called ADHM potential. Minimization of the ADHM potential is equivalent to the ADHM constraint, which should be solved for construction of instantons in the ADHM formalism. Note that since Lagrangian is Hermitian, $\vec{\tau}_{\dot{\beta}}^{\dot{\alpha}} \bar{X}^{\dot{\beta} \alpha} X_{\alpha \dot{\alpha}}$ and $\vec{\tau}_{\dot{\beta}}^{\dot{\alpha}} \bar{w}_{i}^{\dot{\beta}} w_{\dot{\alpha} i}$ are real.

Since our theory is 0+1-dimensional, the gauge field $A_{0}$ is an auxiliary field, and we integrate it out explicitly. The terms including $A_{0}$ in the matrix model action is

$$
S_{A_{0}}=\frac{\lambda N_{c} M_{\mathrm{KK}}}{54 \pi} \int d t\left[\partial_{0} \bar{w}^{\dot{\alpha}}(-i) w_{\dot{\alpha}} A_{0}+i A_{0} \bar{w}^{\dot{\alpha}} \partial_{0} w_{\dot{\alpha}}+\left(A_{0}\right)^{2} \bar{w}^{\dot{\alpha}} w_{\dot{\alpha}}+\frac{54 \pi}{\lambda M_{\mathrm{KK}}} A_{0}\right]
$$

So, the equation of motion for this $A_{0}$, in other words, the Gauss law constraint, is

$$
\frac{54 \pi}{\lambda M_{\mathrm{KK}}}+i\left(\bar{w}_{i}^{\dot{\alpha}} \partial_{0} w_{\dot{\alpha}}^{i}-\partial_{0} \bar{w}_{i}^{\dot{\alpha}} w_{\dot{\alpha}}^{i}\right)+2 \bar{w}_{i}^{\dot{\alpha}} w_{\dot{\alpha}}^{i} A_{0}=0
$$

Then after path-integration over $A_{0}$, we obtain

$$
S_{A_{0}}=\frac{\lambda N_{c}}{54 \pi} M_{\mathrm{KK}} \int d \xi^{0}\left[-\frac{1}{4 \bar{w}_{i}^{\dot{\alpha}} w_{\dot{\alpha}}^{i}}\left(\frac{54 \pi}{\lambda M_{\mathrm{KK}}}+i\left(\bar{w}_{i}^{\dot{\alpha}} \partial_{0} w_{\dot{\alpha}}^{i}-\partial_{0} \bar{w}_{i}^{\dot{\alpha}} w_{\dot{\alpha}}^{i}\right)\right)^{2}\right] .
$$

Using the definition of the momentum conjugate to the field $w$

$$
P_{i}^{\dot{\alpha}} \equiv{\frac{\partial S^{i}}{\partial \dot{w}_{\dot{\alpha}}}}^{i}=\frac{\lambda N_{c} M_{\mathrm{KK}}}{54 \pi}\left[\partial_{0} \bar{w}_{i}^{\dot{\alpha}}-\frac{2}{4 \bar{w}_{j}^{\dot{\gamma}} w_{\dot{\gamma}}^{j}}\left(\frac{54 \pi}{\lambda M_{\mathrm{KK}}}+i\left(\bar{w}_{k}^{\dot{\beta}} \partial_{0} w_{\dot{\beta}}^{k}-\partial_{0} \bar{w}_{k}^{\dot{\beta}} w_{\dot{\beta}}^{k}\right)\right) i \bar{w}_{i}^{\dot{\alpha}}\right]
$$

we obtain the Hamiltonian

$$
\begin{aligned}
H \equiv & P_{i}^{\dot{\alpha}} \dot{w}_{i}^{\dot{\alpha}}+\bar{P}_{\dot{\alpha}}^{i} \dot{\bar{w}}_{\dot{\alpha}}^{i}-L \\
= & \frac{\lambda N_{c} M_{\mathrm{KK}}}{54 \pi}\left[\partial_{0} \bar{w}_{i}^{\dot{\alpha}} \partial_{0} w_{\dot{\alpha}}^{i}+\frac{1}{6} M_{\mathrm{KK}}^{2} \bar{w}_{i}^{\dot{\alpha}} w_{\dot{\alpha}}^{i}\right. \\
& +\frac{\lambda^{2} M_{\mathrm{KK}}^{4}}{3^{6} \pi^{2}}\left[4 w_{1}^{i}\left(w_{2}^{i}\right)^{*} w_{2}^{j}\left(w_{1}^{j}\right)^{*}+\left(w_{1}^{i}\left(w_{1}^{i}\right)^{*}\right)^{2}+\left(w_{2}^{i}\left(w_{2}^{i}\right)^{*}\right)^{2}-2 w_{1}^{i}\left(w_{1}^{i}\right)^{*} w_{2}^{j}\left(w_{2}^{j}\right)^{*}\right] \\
& \left.+\frac{1}{4 \bar{w}_{i}^{\dot{\alpha}} w_{\dot{\alpha}}^{i}}\left(\left(\frac{54 \pi}{\lambda M_{\mathrm{KK}}}\right)^{2}+\left(\bar{w}_{i}^{\dot{\alpha}} \partial_{0} w_{\dot{\alpha}}^{i}-\partial_{0} \bar{w}_{i}^{\dot{\alpha}} w_{\dot{\alpha}}^{i}\right)^{2}\right)\right]
\end{aligned}
$$




\subsection{Single flavor}

Let us minimize the Hamiltonian to find a vacuum of the $k=1$ system. We consider first the case of the single flavor, $N_{f}=1$. We put the following ansatz, ${ }^{10}$

$$
w_{\dot{\alpha}=1}=\rho_{1}, \quad w_{\dot{\alpha}=2}=\rho_{2},
$$

where $\rho$ 's are real constants. Then, the Hamiltonian is

$$
H=\frac{\lambda N_{c} M_{\mathrm{KK}}}{54 \pi}\left[\frac{1}{2}\left(\frac{27 \pi}{\lambda M_{\mathrm{KK}}}\right)^{2} \rho^{-2}+\frac{1}{3} M_{\mathrm{KK}}^{2} \rho^{2}+\frac{4 \lambda^{2} M_{\mathrm{KK}}^{4}}{3^{6} \pi} \rho^{4}\right]
$$

where we have defined $2 \rho^{2} \equiv \rho_{1}^{2}+\rho_{2}^{2}$.

Each term in the Hamiltonian (3.9) has a physical meaning.

- The first term $\propto \rho^{-2}$ is induced by the CS term and the $A_{0}$ path-integration, and it can be interpreted as a self-repulsion of the dyonic instanton in the soliton picture. As described in $[17,18]$, the instanton has an electric charge, so the self-energy should be lowered by expanding the size of the instanton, thus resulting in a negative power in $\rho$.

- The second term is from the mass term of our matrix model, thus comes from the curved spacetime of the background. In terms of the instanton, the location of the instanton along the direction $z$ affects the total mass of the instanton, due to the curved geometry.

- The third term $\propto \rho^{4}$ is from a path-integration over auxiliary $\vec{D}$ fields which for example, yielded the commutator square term in the matrix model action. So this corresponds to the ADHM potential term. For the single flavor, there is no $\mathrm{U}(1)$ instanton except for the small instanton singularity in flat space, and this term ensures it, in the absence of the dyonic coupling and the curved geometry.

The Hamiltonian is minimized at a nonzero $\rho$, but the minimization problem is a nonlinear equation. With a help of the fact that we are working in the large $\lambda$ limit, we reduce the problem to a linear one. We put

$$
\rho=x \lambda^{\alpha} M_{\mathrm{KK}}^{-1}
$$

${ }^{10} \mathrm{~A}$ more general ansatz is,

$$
w_{\dot{\alpha}=1}=\rho_{1} e^{i\left(v_{1} t+s_{1}\right)}, \quad w_{\dot{\alpha}=2}=\rho_{2} e^{i\left(v_{2} t+s_{2}\right)},
$$

where $\rho$ 's, $v$ 's and $s$ 's are real constants. However, this results in the vacuum which is the same as what we will find below in this section. In fact, if we minimize the Hamiltonian $H$ with respect to $v_{1}$ and $v_{2}$, we find $v_{1}=v_{2}$, and the Hamiltonian $H$ is independent of $v$. This is interpreted as a manifestation of the gauge invariance. As a result, we can always choose a gauge $v_{1}=v_{2}=0$. Note that if one choose another gauge, for example, $A_{0}=0$, then the Gauss law constraint (3.3) forces $w$ to have a time-dependence, i.e. $v_{1}=v_{2} \neq 0$ to satisfy the Gauss law constraint (3.3). Simplified ansatz (3.8) is not consistent with a generic gauge choice for $A_{0}$. This is in good contrast to the situation of the ADHM vacuum for supersymmetric Yang-Mills-Chern-Simons instanton studied in [29, 30]. 
where $x$ is a constant coefficient, and $\alpha$ is a constant power. Then, each term in the Hamiltonian (3.9) scales for large $\lambda$ as

$$
\frac{1}{2}\left(\frac{27 \pi}{\lambda M_{\mathrm{KK}}}\right)^{2} \rho^{-2} \sim \lambda^{-2-2 \alpha}, \quad \frac{1}{3} M_{\mathrm{KK}}^{2} \rho^{2} \sim \lambda^{2 \alpha}, \quad \frac{4 \lambda^{2} M_{\mathrm{KK}}^{4}}{3^{6} \pi} \rho^{4} \sim \lambda^{4 \alpha+2} .
$$

In minimizing the Hamiltonian, the first term, which has a negative power of $\rho$, should be balanced with either the second or the third term which has a positive power of $\rho$. If it is with the second term, then from the above $\lambda$-scaling we need to have $-2-2 \alpha=2 \alpha$, thus $\alpha=-1 / 2$. However this value means that the third term has a power larger than the first and the second terms, so leading to an inconsistency. Therefore, we conclude that the minimized Hamiltonian is dominated by a cancellation of the first and the third term. This leads to $-2-2 \alpha=4 \alpha+2$ which is solved as $\alpha=-2 / 3$ at which indeed the second term gives a smaller contribution, which is consistent. For this reason, we can safely ignore the second term at the large $\lambda$, so

$$
H \simeq \frac{\lambda N_{c} M_{\mathrm{KK}}}{54 \pi}\left[\frac{1}{2}\left(\frac{27 \pi}{\lambda M_{\mathrm{KK}}}\right)^{2} \rho^{-2}+\frac{4 \lambda^{2} M_{\mathrm{KK}}^{4}}{3^{6} \pi} \rho^{4}\right] .
$$

Ignoring the second term means that the ADHM-like potential (the third term) is much larger than the curvature scale of the background spacetime. This is natural, since the ADHM-like potential has the scale of the string length, in D-brane effective actions. We will see in the next that for the two flavor case the ADHM-like potential can vanish so that finally the Hamiltonian is minimized by the cancellation of the first and the second terms.

The value of $x$ minimizing this Hamiltonian is computed, as

$$
\rho=2^{-2 / 3} 9 \sqrt{\pi} \lambda^{-2 / 3} M_{\mathrm{KK}}^{-1} .
$$

The minimized value of the Hamiltonian is

$$
H_{\mathrm{min}}=2^{-5 / 3} \lambda^{1 / 3} N_{c} M_{\mathrm{KK}}
$$

\subsection{Two flavors}

Let us consider the more realistic two-flavor case. First, to eliminate the contribution from the ADHM potential term (the third term), we need to satisfy the ADHM constraints, $\vec{\tau}_{\dot{\beta}}^{\dot{\alpha}} \bar{w}_{i}^{\dot{\beta}} w_{\dot{\alpha} i}=0$ for all Pauli matrix directions, or equivalently,

$$
\sum_{i=1}^{N_{f}} w_{\dot{\alpha}=1}^{i}\left(w_{\dot{\alpha}=2}^{i}\right)^{*}=\sum_{i=1}^{N_{f}} w_{\dot{\alpha}=2}^{i}\left(w_{\dot{\alpha}=1}^{i}\right)^{*}=0, \quad \sum_{i=1}^{N_{f}}\left|w_{\dot{\alpha}=1}^{i}\right|^{2}=\sum_{i=1}^{N_{f}}\left|w_{\dot{\alpha}=2}^{i}\right|^{2}
$$

Once this condition is met, the ADHM potential disappears, and the total energy is lowered drastically as the power in $\lambda$ changes. This can be achieved by the following generic choice

$$
w_{\dot{\alpha}}^{i=1}=\left(\begin{array}{l}
\rho \\
0
\end{array}\right)_{\dot{\alpha}}, \quad w_{\dot{\alpha}}^{i=2}=\left(\begin{array}{l}
0 \\
\rho
\end{array}\right)_{\dot{\alpha}} .
$$


Note that this is a generic solution minimizing the ADHM potential, since the condition is invariant under the $\mathrm{U}(2)$ global transformation on the spinor index $\dot{\alpha}$ and the $\mathrm{U}(2)$ flavor symmetry,

$$
w_{\dot{\alpha}}^{i} \rightarrow U_{\dot{\alpha}}^{\dot{\beta}} w_{\dot{\beta}}^{j}\left[U_{\mathrm{f}}^{\dagger}\right]_{j}{ }^{i}
$$

Then the Hamiltonian, after we include $X^{4}$-dependence as well, is

$$
H=\frac{\lambda N_{c} M_{\mathrm{KK}}}{54 \pi}\left[\left(\frac{27 \pi}{\lambda M_{\mathrm{KK}}}\right)^{2} \frac{1}{2 \rho^{2}}+\frac{1}{3} M_{\mathrm{KK}}^{2} \rho^{2}+\frac{2}{3} M_{\mathrm{KK}}^{2}\left(X^{4}\right)^{2}\right] .
$$

This is minimized at

$$
\rho=2^{-1 / 4} 3^{7 / 4} \sqrt{\pi} \lambda^{-1 / 2} M_{\mathrm{KK}}^{-1} .
$$

The minimized value of the Hamiltonian is

$$
H_{\min }=6^{-1 / 2} N_{c} M_{\mathrm{KK}}
$$

This is independent of $\lambda$, thus in the large $\lambda$ limit, we see that $H_{\min }$ for the two flavor case is far smaller than that of the single-flavor case. Recall that the classical mass of the holographic baryon is $\lambda N_{c} M_{\mathrm{KK}} / 27 \pi+H_{\text {min }}$ where the first term comes from the constant part of (2.14).

Here, the variable $\rho$ is nothing but the instanton size in the soliton approach, since we have chosen a correct normalization for this $\rho$, a la ADHM formalism in the flat spacetime. Let us compare our Hamiltonian with the one obtained in the soliton approach, [17, 18], where a potential for moduli of the single instanton solution was computed. The Hamiltonian $[17,18]$ for the instanton size modulus $\rho$ and the instanton location $Z$ along the $x^{4}$ direction is

$$
H_{\text {soliton }}=\frac{\lambda N_{c} M_{\mathrm{KK}}}{54 \pi}\left[\frac{2 \cdot 3^{6} \pi^{2}}{5\left(\lambda M_{\mathrm{KK}}\right)^{2}} \frac{1}{\rho^{2}}+\frac{1}{3} M_{\mathrm{KK}}^{2} \rho^{2}+\frac{2}{3} M_{\mathrm{KK}}^{2}\left(X^{4}\right)^{2}\right],
$$

again without the rest mass term $\lambda N_{c} M_{\mathrm{KK}} / 27 \pi$. We first note that the quadratic terms in $X^{4}$ and in $\rho$ coincide with ours. The coincidence of the $X^{4}$ mass term is nontrivial, while the one for $\rho$ is not accidental, since we have computed the $\rho$ mass term in (2.1) by resorting to the soliton picture in the supersymmetric limit. In some sense, they are first order terms whose evaluation used the zero-th order solution. The real comparison is with the first terms which are proportional to $1 / \lambda^{2}$ (times the naive baryon mass computed from $D 4^{\prime}$ tension); the two are structurally identical but the matrix model result is larger than the soliton result by a factor of $5 / 4$.

In the soliton picture, this term arises as the five-dimensional Coulomb energy associated with $\mathrm{U}(1)$ baryon charge; the latter is, due to the holographic map, a gauge charge of real gauge field on $D 8$. In the matrix model, it comes from integrating out the (nondynamical) $A_{0}$ gauge field on $D 4^{\prime}$. Either way, it comes from a quadrature of an excitation field of order $1 / \lambda$, which suggests that, in the supersymmetric limit of very small instanton, 
the Coulombic energy captures the deviation from the zero-th order "instanton = ADHM" configuration more effectively. We suspect that this explains the numerical difference. At any rate, $5 / 4$ is fairly close to 1 , implying that the difference between the two approaches is relatively minor. Since the two such models in general live in two vastly different validity regions, respectively, one could have expected a larger difference. It is the presence of approximate supersymmetry in distance scale from $1 / M_{K K}$ down to $l_{s}^{\text {eff }}$ and below that give us this relative stability. The baryon physics of one does not deviate a lot from the other.

\subsection{Quantization}

With this result on the matrix model vacuum in mind, we can now quantize the small fluctuations of the $k=1 N_{f}=2$ matrix model. This spectrum should correspond to the baryon spectrum.

As we have seen, what is different from [18] is just the coefficient in front of the $1 / \rho^{2}$ term in the Hamiltonian. So, we can just track the difference in the computations of [18] and find the spectrum of our matrix model. This difference reflects in the constant $Q$ in [18], which is now multiplied by $5 / 4$ in our case. Then the mass formula for the baryon excitation is

$$
M=M_{0}+\sqrt{\frac{(l+1)^{2}}{6}+\frac{N_{c}^{2}}{6}}+\frac{2\left(n_{\rho}+n_{Z}\right)+2}{\sqrt{6}} .
$$

Here $n_{\rho}, n_{Z}=0,1,2, \cdots$ and $l=I / 2=J / 2$ with spin $J$ and isospin $I$. The difference from [18] is just the coefficient of $N_{c}^{2}$ in this expression. $M_{0}$ is the mass of the D4'-brane which is equal to the first term in (2.14), $\lambda N_{c} M_{\mathrm{KK}} / 27 \pi$.

This formula in particular means that we can obtain

$$
M_{l=3}-M_{l=1}=0.5693 M_{\mathrm{KK}} .
$$

If we use $M_{\mathrm{KK}}=945[\mathrm{MeV}]$ which is fit by the $\rho$ meson mass, we obtain

$$
M_{\Delta}-M_{N / P}=540[\mathrm{MeV}] .
$$

This is larger than the experimental value $292[\mathrm{MeV}]$. The situation is similar to the soliton approach [18] which gave the value $569[\mathrm{MeV}]$.

\subsection{Meson couplings}

As we noted toward the end of subsection (3.3), the energetics of $\rho$ here differ quantitatively from the previous estimate based on the instanton soliton picture in D8-brane gauge theory. One consequence is that the classical value of $\rho$, which is the holographic size of the nucleon, is slightly larger that its previous estimates in refs. $[17,18]$. Denoting the latter by $\rho_{\text {soliton }}$, we found

$$
\rho^{2}=\sqrt{\frac{5}{4}} \rho_{\text {soliton }}^{2}
$$

This new estimate modified the baryon spectra as we just saw above, but it should also affect couplings to mesons. 
This can be seen most clearly from the effective action approach $[17,19]$, where a treelevel effective action in the five-dimensional bulk captures nucleon $\mathcal{N}$ coupled to mesons

$$
\int\left[-i \overline{\mathcal{N}} \gamma^{m}\left(\partial_{m}-i \mathcal{A}_{m}^{\mathrm{U}(2)}\right) \mathcal{N}-i m_{\mathcal{N}} \overline{\mathcal{N}} \mathcal{N}+\frac{2 \pi^{2} \rho_{\text {soliton }}^{2}}{3 e^{2}} \overline{\mathcal{N}} \gamma^{m n} F_{m n}^{\mathrm{SU}(2)} \mathcal{N}\right]
$$

$m_{\mathcal{N}}$ and $e^{2}$ are known functions of the holographic $Z$-coordinate, determined by the dual geometry. Actual four-dimensional nucleon is the lowest lying mode of five-dimensional Dirac field $\mathcal{N}$ (here, denoted by the same symbol in abuse of notation), while the infinite tower of mesons are embedded into the five-dimensional flavor gauge field $\mathcal{A}^{\mathrm{U}(2)}=A^{\mathrm{U}(1)}+$ $A^{\mathrm{SU}(2)}$ as in ref. [11]. Note that the size parameter $\rho_{\text {soliton }}^{2}$ appears explicitly only once, in the last term, so the holographic size of the nucleon will affect couplings to $\mathrm{SU}(2)$ iso-triplet meson coupling with specific chiral or tensor structures only.

Previous estimates of the meson-nucleon-nucleon couplings were based on the instanton soliton viewpoint and may not be completely compatible with our new matrix model. Nevertheless, we note that these couplings were read-off entirely from the long distance gauge field configuration associated with the baryon [17, 19], which has to be the case since the Compton wavelength of mesons at $\sim 1 / M_{\mathrm{KK}}$ are much larger than the size of the soliton core $\rho_{\text {soliton }} \sim 1 / M_{K K} \sqrt{\lambda}$. This suggests that the derivations of meson-baryon coupling are generally safe from short-distance physics. The one place where this reasoning can go wrong is the size of the soliton itself, whose estimate relies heavily on short distance physics and which in turn affect the long distance magnetic field of the soliton. Thus, we expect that the effect of the latter manifests in the meson-baryon couplings via the single quantity, $\rho^{2}$.

We can classify the cubic meson-nucleon-nucleon couplings from (3.26) into two classes. The first class consist of those whose leading large $N_{c}$ behaviors originate from the minimal coupling to $\mathcal{A}^{\mathrm{U}(2)}$. This includes dimensions-four couplings to vector mesons, and dimension-four couplings to all iso-singlet mesons: vector, axial vector, or pseudo-scalar. With the exception of those to iso-singlet vectors, this class of couplings are subleading in $1 / N_{c}$. Let us collectively denote them as $g^{(I)}$.

The second class, $g^{(I I)}$, have the leading $N_{c}$ contributions arising from the last term of (3.26) and thus proportional to $\rho^{2}$. This class consists of coupling to iso-triplet Goldstone boson (namely pions) [17], minimal couplings to all iso-triplet axial vector mesons [19], and dimension-five tensor couplings to all iso-triplet vector mesons [23]. When we replace $\rho_{\text {soliton }}^{2}$ by $\rho^{2}$, we therefore find

$$
g^{(I)}=g_{\text {soliton }}^{(I)}, \quad g^{(I I)}=\sqrt{\frac{5}{4}} g_{\text {soliton }}^{(I I)} .
$$

Numerically, the latter represents about $12 \%$ increase for $g^{(I I)}$ 's.

Translating to quantities more directly related to data, we have for example the charge form factor and the isospin-independent part of nucleon-nucleon repulsive core unaffected, whereas the magnetic form factor (thus the anomalous magnetic moments also) and the isospin-dependent part of nucleon-nucleon potential would be increased by factor of $5 / 4$ in the large $N_{c}$ limit. See the next section for a direct matrix model computation of the nucleon-nucleon potential at short distance, which confirms this expectation. 
Whether or not such a shift is beneficial in reproducing QCD with $N_{c}=3$ is unclear and needs to be studied more. We would know the answer only after we have classified and computed subleading corrections in this D4-D8 model. Regardless, we note that, purely within the context of studying baryons in the D4-D8 model, this represents a significant change to the leading $1 / N_{c}$ computation (and not a subleading correction) which could have not been obtained by any other method we know of.

\section{Two-body baryon interaction}

The baryon interaction at short distance can be obtained by classically integrating out $A_{0}$, as in the case of the single baryon. Now, with two baryons, we have the matrices charged under the $\mathrm{U}(2)$, so, in particular, $A_{0}$ has four components,

$$
A_{0}=A_{0}^{0} \mathbf{1}_{2 \times 2}+A_{0}^{1} \tau^{1}+A_{0}^{2} \tau^{2}+A_{0}^{3} \tau^{3} .
$$

Recall that, in the previous section, solving for the equation of motion of the overall $\mathrm{U}(1)$ gauge field gave the $1 / \rho^{2}$ potential for individual baryon. Now we are interested in integrating non-Abelian part of $A_{0}$ as well, which should generate interaction energy for a pair of baryons.

Note that $A_{0}^{a}(a=1,2,3)$ does not show up in our CS term in (2.1), because the latter contains $\operatorname{tr} A_{0}$ only. Terms including $A_{0}^{a}$ appear in the kinetic term of $X$ and that of $w$.

\subsection{Two-baryon configuration}

In order to evaluate the action, first we fix the vacuum of the ADHM potential in (2.1).

\subsubsection{Single flavor}

The classical single baryon configuration is specified by the vacuum configuration of $w$. In section 3.2 , we obtained the classical vacuum of the matrix model as

$$
w_{\dot{\alpha}}=U\left(\begin{array}{l}
1 \\
0
\end{array}\right)_{\dot{\alpha}} \rho
$$

where $U$ is a $2 \times 2$ unitary matrix. This representation is equivalent to having $\rho_{1}, \rho_{2}$ in the notation of section 3.2. This unitary matrix comes from the global symmetry (3.17). Now we have two baryons, so the baryons are specified by two unitary matrices,

$$
w_{\dot{\alpha}}^{i=1}=U^{(1)}\left(\begin{array}{l}
1 \\
0
\end{array}\right)_{\dot{\alpha}} \rho, \quad w_{\dot{\alpha}}^{i=2}=U^{(2)}\left(\begin{array}{l}
1 \\
0
\end{array}\right)_{\dot{\alpha}} \rho .
$$

One can work out the baryon interaction potential with this, but we will perform the computation only in the 2-flavor case in the following, since it is realistic. 


\subsubsection{Two flavors}

For the two-flavor case, the vacuum configuration of the potential is given by the integration of the $\mathrm{U}(k)$-adjoint field $\vec{D}_{A B}$ in the matrix model (2.1), which is the ADHM constraints,

$$
\vec{\tau}_{\dot{\beta}}^{\dot{\alpha}}\left(\bar{X}^{\dot{\beta} \alpha} X_{\alpha \dot{\alpha}}+\bar{w}_{i}^{\dot{\beta}} w_{\dot{\alpha} i}\right)_{B A}=0 .
$$

Here we explicitly write the baryon index $A, B=1, \ldots, k$. In this section we treat two instantons so $k=2$. The generic ADHM configuration satisfying this equation is nothing but the ADHM data of two YM instantons. It is given by

$$
\begin{aligned}
X_{M} & =\tau^{3} \frac{r_{M}}{2}+\tau^{1} Y_{M}, \\
w_{\dot{\alpha} i}^{A=1} & =U_{\dot{\alpha} i}^{(A=1)} \rho_{1}, \quad w_{\dot{\alpha} i}^{A=2}=U_{\dot{\alpha} i}^{(A=2)} \rho_{2},
\end{aligned}
$$

where the locations of the two baryons are given by the diagonal entries in $X_{M}$ so that $r_{M}$ is the inter-baryon distance, and

$$
Y_{M} \equiv-\frac{\rho_{1} \rho_{2}}{4\left(r_{P}\right)^{2}} \operatorname{tr}\left[\bar{\sigma}_{M} r_{N} \sigma_{N}\left(\left(U^{(1)}\right)^{\dagger} U^{(2)}-\left(U^{(2)}\right)^{\dagger} U^{(1)}\right)\right] .
$$

Here $U^{(1)}$ and $U^{(2)}$ are $\mathrm{SU}(2)$ matrices which denote the moduli parameters of each baryon, and $\sigma_{M} \equiv(i \vec{\tau}, 1), \bar{\sigma}_{M} \equiv(-i \vec{\tau}, 1)$.

This ADHM data was explicitly used in the soliton approach [22]. In terms of the YM instanton, these degrees of freedom are gauge rotations of the "flavor" gauge group, and after the quantization, they become the spin and the isospin of each baryon. They can be written by real unit vectors $a_{M}^{(1)}$ and $a_{M}^{(2)}$ as

$$
U^{(1)}=i a_{i}^{(1)} \tau^{i}+a_{4}^{(1)} \mathbf{1}_{2 \times 2}, \quad U^{(2)}=i a_{i}^{(2)} \tau^{i}+a_{4}^{(2)} \mathbf{1}_{2 \times 2},
$$

with $\left(a_{4}^{(1)}\right)^{2}+\left(a_{i}^{(1)}\right)^{2}=1,\left(a_{4}^{(2)}\right)^{2}+\left(a_{i}^{(2)}\right)^{2}=1$. This is the correspondence to the notation of [18]. Using this, we obtain expressions which will be useful later, ${ }^{11}$

$$
\begin{aligned}
r_{M} Y_{M} & =0 \\
Y_{M} Y_{M} & =-\frac{\rho_{1}^{2} \rho_{2}^{2}}{8\left(r_{M}\right)^{2}} \operatorname{tr}\left[\left(U^{(1) \dagger} U^{(2)}-U^{(2) \dagger} U^{(1)}\right)^{2}\right]=\frac{\rho_{1}^{2} \rho_{2}^{2}}{4\left(r_{M}\right)^{2}}\left(1-\left(a_{M}^{(1)} a_{M}^{(2)}\right)^{2}\right), \\
\operatorname{tr}\left[U^{(1) \dagger} U^{(2)}\right] & =\operatorname{tr}\left[U^{(2) \dagger} U^{(1)}\right]=2 a_{M}^{(1)} a_{M}^{(2)} .
\end{aligned}
$$

\subsection{Baryon interaction potential}

We shall integrate out the matrix $A_{0}$ and compute the two-baryon interaction potential. For integrating out the $\mathrm{SU}(2)$ components of $A_{0}$, we first write down all the terms including those components in the matrix model action (2.1). First,

$$
\operatorname{tr}\left(D_{0} X^{M}\right)^{2}=2\left(\left(A_{0}^{1}\right)^{2} r_{M}^{2}+\left(A_{0}^{2}\right)^{2}\left(r_{M}^{2}+4 Y_{M}^{2}\right)+4\left(A_{0}^{3}\right)^{2} Y_{M}^{2}\right)-8 A_{0}^{1} A_{0}^{3} r_{M} Y_{M} .
$$

\footnotetext{
${ }^{11}$ To obtain the first equality of (4.11), we have used the following formula for unifying the double trace:

$$
\left(\operatorname{tr}\left[\bar{\sigma}_{M}\left(b_{0}+i b_{i} \tau^{i}\right)\right]\right)^{2}=2 \operatorname{tr}\left[\left(b^{0}+i b^{i} \tau^{i}\right)\left(b^{0}-i b^{j} \tau^{j}\right)\right] .
$$

Then, using $\left(a_{4}^{(1)}\right)^{2}+\left(a_{i}^{(1)}\right)^{2}=1$ and $\left(a_{4}^{(2)}\right)^{2}+\left(a_{i}^{(2)}\right)^{2}=1$, the second equality of (4.11) follows.
} 
Here we used only (4.5). But if we further use the explicit expression (4.7) and the vacuum for $w(4.6)$, we obtain (4.10), so the last term in (4.13) vanishes. Next, the $w$ kinetic term is

$$
\begin{aligned}
\operatorname{tr} D_{0} \bar{w}_{i}^{\dot{\alpha}} D_{0} w_{\dot{\alpha} i}= & 2\left(\rho_{1}^{2}+\rho_{2}^{2}\right)\left(\left(A_{0}^{0}\right)^{2}+\left(A_{0}^{1}\right)^{2}+\left(A_{0}^{2}\right)^{2}+\left(A_{0}^{3}\right)^{2}\right) \\
& +4 \rho_{1} \rho_{2} A_{0}^{0} A_{0}^{1} \operatorname{tr}\left[U^{(1) \dagger} U^{(2)}\right]+4\left(\rho_{1}^{2}-\rho_{2}^{2}\right) A_{0}^{0} A_{0}^{3} .
\end{aligned}
$$

In these kinetic terms, the component $A_{0}^{2}$ appears only as a form $\left(A_{0}^{2}\right)^{2}$. Thus we can minimize it independently with $A_{0}^{2}=0$, meaning that we can just ignore the component $A_{0}^{2}$. So, the total kinetic action plus the Chern-Simons term is

$$
\begin{aligned}
& \frac{\lambda N_{c} M_{\mathrm{KK}}}{54 \pi} \int d t \operatorname{tr}\left[\left(D_{0} X^{M}\right)^{2}+D_{0} \bar{w}_{i}^{\dot{\alpha}} D_{0} w_{\dot{\alpha} i}\right]+N_{c} \int d t \operatorname{tr} A_{0} \\
& =\frac{\lambda N_{c} M_{\mathrm{KK}}}{54 \pi} \int d t\left[2\left(A_{0}^{1}\right)^{2} r_{M}^{2}+8\left(A_{0}^{3}\right)^{2} Y_{M}^{2}+2\left(\rho_{1}^{2}+\rho_{2}^{2}\right)\left(\left(A_{0}^{0}\right)^{2}+\left(A_{0}^{1}\right)^{2}+\left(A_{0}^{3}\right)^{2}\right)\right. \\
& \left.\quad+4 \rho_{1} \rho_{2} A_{0}^{0} A_{0}^{1} \operatorname{tr}\left[U^{(1) \dagger} U^{(2)}\right]+4\left(\rho_{1}^{2}-\rho_{2}^{2}\right) A_{0}^{0} A_{0}^{3}+\frac{108 \pi}{\lambda M_{\mathrm{KK}}} A_{0}^{0}\right]
\end{aligned}
$$

As the action is quadratic in the remaining components of $A_{0}$, it is straightforward to integrate them out by diagonalizing the interaction terms. The resultant baryon interaction potential $V$ is determined from $\int d t V=-S_{\text {on-shell }}$ as

$$
V=\frac{27 \pi N_{c}}{\lambda M_{\mathrm{KK}}} \frac{1}{\rho_{1}^{2} \rho_{2}^{2}} \frac{\left(\left(r_{M}\right)^{2}+\rho_{1}^{2}+\rho_{2}^{2}\right)\left(4\left(r_{M}\right)^{2}\left(\rho_{1}^{2}+\rho_{2}^{2}\right)-u \rho_{1}^{2} \rho_{2}^{2}\right)}{16\left(\left(r_{M}\right)^{2}\right)^{2}-5 u\left(r_{M}\right)^{2}\left(\rho_{1}^{2}+\rho_{2}^{2}\right)-u\left(\rho_{1}^{4}+\rho_{2}^{4}-(u+2) \rho_{1}^{2} \rho_{2}^{2}\right)},
$$

where $u \equiv\left(\operatorname{tr}\left[U^{(1) \dagger} U^{(2)}\right]\right)^{2}-4$.

In addition to this potential $V$, we have another baryon interaction potential which comes from the mass term of $X^{4}$ in the matrix model action (2.1). It is easily evaluated as

$$
\frac{\lambda N_{c} M_{\mathrm{KK}}}{54 \pi} \cdot \frac{2}{3} M_{\mathrm{KK}}^{2} \operatorname{tr}\left(X^{4}\right)^{2}=\frac{\lambda N_{c}}{81 \pi} M_{\mathrm{KK}}^{3}\left(\left(r_{4}\right)^{2} / 2+2\left(Y_{4}\right)^{2}\right) .
$$

Here $\left(r_{4}\right)^{2}$ term is the mass term in the single-baryon Hamiltonian, so it does not contribute to the two-baryon interaction. On the other hand, the off-diagonal element $Y^{4}$ is intrinsically the interaction between the baryons. The definition (4.7) is computed as

$$
Y_{4}=-\frac{\rho_{1} \rho_{2}}{2 r_{M}^{2}} r_{i} \operatorname{tr}\left[i \tau^{i}\left(U^{(1)}\right)^{\dagger} U^{(2)}\right]
$$

where $i=1,2,3$, so we can write the potential energy explicitly as

$$
\frac{\lambda N_{c} M_{\mathrm{KK}}^{3}}{162 \pi}\left[\left(r_{4}\right)^{2}+\frac{\rho_{1}^{2} \rho_{2}^{2}}{\left(r_{M}^{2}\right)^{2}}\left(r_{i} \operatorname{tr}\left[i \tau^{i}\left(U^{(1)}\right)^{\dagger} U^{(2)}\right]\right)^{2}\right] .
$$

Therefore, in total, the two-baryon interaction Hamiltonian is given by

$$
\begin{aligned}
V= & \frac{27 \pi N_{c}}{\lambda M_{\mathrm{KK}}} \frac{1}{\rho_{1}^{2} \rho_{2}^{2}} \frac{\left(\left(r_{M}\right)^{2}+\rho_{1}^{2}+\rho_{2}^{2}\right)\left(4\left(r_{M}\right)^{2}\left(\rho_{1}^{2}+\rho_{2}^{2}\right)-u \rho_{1}^{2} \rho_{2}^{2}\right)}{16\left(\left(r_{M}\right)^{2}\right)^{2}-5 u\left(r_{M}\right)^{2}\left(\rho_{1}^{2}+\rho_{2}^{2}\right)-u\left(\rho_{1}^{4}+\rho_{2}^{4}-(u+2) \rho_{1}^{2} \rho_{2}^{2}\right)} \\
& +\frac{\lambda N_{c} M_{\mathrm{KK}}^{3}}{162 \pi} \frac{\rho_{1}^{2} \rho_{2}^{2}}{\left(r_{M}^{2}\right)^{2}}\left(r_{j} \operatorname{tr}\left[i \tau^{j}\left(U^{(1)}\right)^{\dagger} U^{(2)}\right]\right)^{2}-\frac{27 \pi N_{c}}{4 \lambda M_{\mathrm{KK}}}\left(\frac{1}{\rho_{1}^{2}}+\frac{1}{\rho_{2}^{2}}\right),
\end{aligned}
$$


where $u \equiv\left(\operatorname{tr}\left[U^{(1) \dagger} U^{(2)}\right]\right)^{2}-4$. The last term is the subtraction of the single-baryon Hamiltonians (the first term in (3.18), while the second term (mass term) in (3.18) cancelled already).

Next, we shall evaluate this potential for a given quantum state of the two baryons, and show that there is a universal repulsive core for any baryon state.

\subsection{Universal repulsive core}

First notice that in the classical limit, i.e. $N_{c} \rightarrow \infty$, the expectation value of any function $\left\langle f\left(\rho_{1}, \rho_{2}\right)\right\rangle$ with any given quantum state of the baryons approaches the classical value $f\left(\rho_{1}=\rho, \rho_{2}=\rho\right)$ where the classical $\rho$ is given by (3.19). The equivalence between $N_{c} \rightarrow \infty$ limit and $\hbar \rightarrow 0$ limit is due to the fact that our matrix action (2.1) has an overall factor $N_{c}$. Since any deviation from the classical value is $\mathcal{O}\left(1 / N_{c}^{2}\right)$, while we are keeping only leading terms in the large $N_{c}$ expansion, we in effect just need to put $\rho_{1}=\rho_{2}(\equiv \rho)$ in our potential (4.20). ${ }^{12}$ We find

$$
\begin{aligned}
V= & \frac{27 \pi N_{c}}{4 \lambda M_{\mathrm{KK}}} \frac{\left(\operatorname{tr}\left[U^{(1) \dagger} U^{(2)}\right]\right)^{2}}{\left(r_{M}\right)^{2}+2 \rho^{2}-\frac{1}{2}\left(\operatorname{tr}\left[U^{(1) \dagger} U^{(2)}\right]\right)^{2} \rho^{2}} \\
& +\frac{\lambda N_{c} M_{\mathrm{KK}}^{3}}{162 \pi} \frac{\rho^{4}}{\left(r_{M}^{2}\right)^{2}}\left(r_{j} \operatorname{tr}\left[i \tau^{j} U^{(1) \dagger} U^{(2)}\right]\right)^{2} .
\end{aligned}
$$

This is positive semi-definite. In fact, we can show that our potential (4.21) never vanish. In order to see (4.21) never vanish, note that it could vanish if and only if $\operatorname{tr}\left[U^{(1) \dagger} U^{(2)}\right]=0$ and $\operatorname{tr}\left[i \tau^{i} U^{(1) \dagger} U^{(2)}\right]=0$ for any $i$. However the latter condition implies $U^{(1) \dagger} U^{(2)} \propto$ $\mathbf{1}_{2 \times 2}$, which contradicts with the former, therefore this is impossible. In this way, we see that (4.21) is positive definite and as a result, there is a universal repulsive potential (core) for any choice of two-baryon quantum states.

If we expand (4.20) for $\left(r_{M}\right)^{2} \gg \rho^{2}$, we obtain a leading term

$$
\begin{aligned}
V= & \frac{27 \pi N_{c}}{64 \lambda M_{\mathrm{KK}}} \frac{1}{\left(r_{M}\right)^{2}}\left(8+6\left(\operatorname{tr}\left[U^{(1) \dagger} U^{(2)}\right]\right)^{2}+\left(-4+5\left(\operatorname{tr}\left[U^{(1) \dagger} U^{(2)}\right]\right)^{2}\right)\left(\frac{\rho_{2}^{2}}{\rho_{1}^{2}}+\frac{\rho_{1}^{2}}{\rho_{2}^{2}}\right)\right) \\
& +\frac{\lambda N_{c} M_{\mathrm{KK}}^{3}}{162 \pi} \frac{\rho_{1}^{2} \rho_{2}^{2}}{\left(r_{M}^{2}\right)^{2}}\left(r_{j} \operatorname{tr}\left[i \tau^{j}\left(U^{(1)}\right)^{\dagger} U^{(2)}\right]\right)^{2} .
\end{aligned}
$$

For the large $N_{c}$, again we can put $\rho_{1}=\rho_{2}=\rho$ and obtain

$$
V=\frac{27 \pi N_{c}}{4 \lambda M_{\mathrm{KK}}}\left(\operatorname{tr}\left[U^{(1) \dagger} U^{(2)}\right]\right)^{2} \frac{1}{\left(r_{M}\right)^{2}}+\frac{\lambda N_{c} M_{\mathrm{KK}}^{3}}{162 \pi} \frac{\rho^{4}}{\left(r_{M}^{2}\right)^{2}}\left(r_{j} \operatorname{tr}\left[i \tau^{j} U^{(1) \dagger} U^{(2)}\right]\right)^{2} .
$$

Again, we see the repulsive core. The universal repulsive potential scales as $1 / r^{2}$ at $r \gg \rho$ where $r$ is the baryon separation. ${ }^{13}$

\footnotetext{
${ }^{12}$ This argument is valid as long as the classical value does not vanish and the wave function localizes for $N_{c} \rightarrow \infty$, which is in fact the present case for $\rho$ and $X^{4}$. As for the spin/isospin encoded in $U^{(1)}$ and $U^{(2)}$ we cannot take the classical value as the wave function is not localized in the group space.

${ }^{13}$ Although the vector $r_{M}$ is in 4 spatial dimensions, once we take the VEV with the wave function of $X^{4}$, it reduces to a 3 -dimensional vector $r_{i}(i=1,2,3)$. This is again because the leading term in the $1 / N_{c}$ expansion is a classical value of $X^{4}$ which is zero.
} 
Let us consider the vacuum expectation value of the Hamiltonian (4.23). The quantum state of the two baryons is specified by $\left(\vec{I}_{i}, \vec{J}_{i}, n_{\rho}^{(1)}, n_{Z}^{(i)}\right)$ with $i=1,2$ which labels the two baryons. $\vec{I}(\vec{J})$ is the isospin (spin) of the baryon, while $n_{\rho}$ and $n_{Z}$ are labels for excited baryon states [18]. Explicit wave functions are given in [18]. For nucleons $(|\vec{I}|=|\vec{J}|=1 / 2)$, the spin/isospin wave functions are

$$
\frac{1}{\pi}\left(\tau^{2} U\right)_{I J}=\left(\begin{array}{ll}
|p \uparrow\rangle & |p \downarrow\rangle \\
|n \uparrow\rangle & |n \downarrow\rangle
\end{array}\right)_{I J}
$$

for each nucleon, so for our two-nucleon case the wave function is

$$
\frac{1}{\pi^{2}}\left(\tau^{2} U^{(1)}\right)_{I_{1} J_{1}}\left(\tau^{2} U^{(2)}\right)_{I_{2} J_{2}}
$$

where $\left(I_{1}, J_{1}, I_{2}, J_{2}\right)$ are the third components of the isospins and the spins for the nucleons, thus take values $\pm 1 / 2$.

Now we obtain an explicit repulsive core for nucleons. At the leading order in $1 / N_{c}$, we can simply take the classical values for $\rho$ and $X^{4}$. As for the spin/isospin part, we can use the formulas given by (4.9), (4.11), and (4.18) of [22],

$$
\begin{gathered}
\left\langle\left(\operatorname{tr}\left[U^{(1) \dagger} U^{(2)}\right]\right)^{2}\right\rangle_{I_{1}, J_{1}, I_{2}, J_{2}}=1+\frac{16}{9} I_{1}^{i} I_{2}^{i} J_{1}^{j} J_{2}^{j} \\
\left\langle\operatorname{tr}\left[i \tau^{i} U^{(1) \dagger} U^{(2)}\right] \operatorname{tr}\left[i \tau^{j} U^{(1) \dagger} U^{(2)}\right]\right\rangle_{I_{1}, J_{1}, I_{2}, J_{2}}=\delta^{i j}+\frac{16}{9} I_{1}^{k} I_{2}^{k}\left(J_{1}^{i} J_{2}^{j}+J_{2}^{j} J_{1}^{i}-\delta^{i j} J_{1}^{k} J_{2}^{k}\right) .
\end{gathered}
$$

These formulas are for a given isospin $I_{A}^{i}$ and spin $J_{A}^{i}$ for the nucleon labeled as $A=1,2$. Finally, the vacuum expectation value of the potential (4.23) gives the central and the tensor forces,

$$
\langle V\rangle_{I_{1}, J_{1}, I_{2}, J_{2}}=V_{\mathrm{C}}(\vec{r})+S_{12} V_{\mathrm{T}}(\vec{r})
$$

with the standard definition $S_{12} \equiv 12 J_{1}^{i} \hat{r}_{i} J_{2}^{j} \hat{r}_{j}-4 J_{1}^{i} J_{2}^{i}\left(\right.$ with $\left.\hat{r}_{i} \equiv r_{i} /|r|\right)$, where

$$
\begin{aligned}
& V_{\mathrm{C}}(\vec{r})=\pi\left(\frac{3^{3}}{2}+8 I_{1}^{i} I_{2}^{i} J_{1}^{j} J_{2}^{j}\right) \frac{N_{c}}{\lambda M_{\mathrm{KK}}} \frac{1}{r^{2}}, \\
& V_{\mathrm{T}}(\vec{r})=2 \pi I_{1}^{i} I_{2}^{i} \frac{N_{c}}{\lambda M_{\mathrm{KK}}} \frac{1}{r^{2}} .
\end{aligned}
$$

This is the short-distance nuclear force obtained from our matrix model. We find there is a repulsive core of nucleons. The repulsive potential scales as $1 / r^{2}$ for the inter-nucleon distance $r$, which is a property peculiar to the holographic model, as noted in [22]. ${ }^{14}$

\footnotetext{
${ }^{14}$ The nucleon-nucleon potential normally consists of two different regimes. One is the long distance regime of order $1 / M_{\mathrm{KK}}$ and beyond [23], where the four-dimensional exchanges of relatively light mesons, including pions of course, dominate nucleon interaction and induces attractive forces. The other is the repulsive core at short distances. One might naively think that the latter is not too relevant for formation of nuclei for example. However, nuclei are finely balanced systems with very small binding energy, and at least numerically seem sensitive to the details of the short distance repulsive core.
} 
Finally let us compare this result with the soliton approach [22] in which the twobaryon interaction potential $V^{\text {soliton }}(\vec{r})=V_{\mathrm{C}}^{\text {soliton }}(\vec{r})+S_{12} V_{\mathrm{T}}^{\text {soliton }}(\vec{r})$ was computed as,

$$
\begin{aligned}
V_{\mathrm{C}}^{\text {soliton }}(\vec{r}) & =\pi\left(\frac{3^{3}}{2}+\frac{32}{5} I_{1}^{i} I_{2}^{i} J_{1}^{j} J_{2}^{j}\right) \frac{N_{c}}{\lambda M_{\mathrm{KK}}} \frac{1}{r^{2}}, \\
V_{\mathrm{T}}^{\text {soliton }}(\vec{r}) & =\frac{8 \pi}{5} I_{1}^{i} I_{2}^{i} \frac{N_{c}}{\lambda M_{\mathrm{KK}}} \frac{1}{r^{2}} .
\end{aligned}
$$

Compared to our (4.28) and (4.29), we can see that the structure of the core is the same. ${ }^{15}$ Furthermore, the numerical coefficients are only different by factor $5 / 4$ for the term proportional to $I_{1}^{i} I_{2}^{i} J_{1}^{j} J_{2}^{j}$ in $V_{\mathrm{C}}^{\text {soliton }}(\vec{r})$ and for the term $V_{\mathrm{T}}^{\text {soliton }}(\vec{r})$. One can see easily that the difference in the last coefficient by factor $5 / 4$ is due to the relation (3.25). More generally, recall that in the large $N_{c}$ one-boson exchange picture of nucleon-nucleon potential [23], the latter two isospin-dependent structures are known to arise only from the second class of couplings, $g^{(I I)}$, of section 3.5. It is the coupling squared that enter the potential, which explains the factor $5 / 4$ increase relative to the soliton model. The isospin-independent central term arises, again in the large $N_{c}$, from iso-singlet vector exchanges, couplings for which belong to the first class, $g^{(I)}$, which explains the agreement.

It is encouraging that the soliton approach and our matrix approach give qualitatively the same, and also quantitatively similar result here as well. The large $N_{c}$ computation is relatively stable in interpolating short distance and long distance regime. At the same time, numerically the increase is hardly negligible and may yet prove to be a big difference in the end, when we have a good control over subleading $1 / N_{c}$ and $1 / \lambda$ corrections in the model. Whether or not this will favor the current D4-D8 model in simulating QCD is unclear for now, however, since some of known subleading $1 / N_{c}$ corrections appear at comparable magnitude if $N_{c}=3$ is used and it is not known how to catalog all such corrections.

Before closing, we would like to make comments on some of existing computations of nucleon-nucleon potential. First of all, we again emphasize that we only computed the repulsive core at very short distance. As we mentioned above, this short-distance repulsive core was computed by the solitonic methods in ref. [22] and also in ref. [36], which differ from our result only by a numerical factor. The fact that there is a universal $1 / r^{2}$ behavior is a clear and unambiguous prediction of holography and needs to be compared against other less speculative approaches, possibly via lattice QCD simulations or by experiments.

Of more practical interest to most of nuclear community would be the long-distance behavior, which should include an attractive iso-singlet channel. From the holographic viewpoint of D4-D8 model, this long-distance behavior was found in ref. [23] where the authors computed one-boson exchange potential, with coupling constants computed precisely using the solitonic picture of the baryon, and indeed found the long-distance attractive potential with right qualitative behaviors, such as allowing deuteron wavefunction nu-

\footnotetext{
${ }^{15} \mathrm{It}$ is also interesting to note that, at the stage of the Hamiltonian, the second term of (4.20) completely coincides with what is called $H_{1}^{(\mathrm{SU}(2))}$ in $[22]$ in its structure. This part basically gives the tensor force. The difference in coefficients in the tensor forces are due to the relation (3.25). Nevertheless, since $H_{1}^{(\mathrm{SU}(2))}$ came in [22] from a generalized Osborn's formula, it is quite interesting that our matrix model can reproduce quite easily the formula.
} 
merically [37]. Our matrix model, in its current form, cannot address this long distance behavior, unfortunately.

There exist extensive literatures on the matter of nucleon-nucleon potential, which are too numerous and too diverse to list. See for instance ref. [38] for computations based on the chiral perturbation theory. In the face of such extensive previous work, one may ask why we should again try to compute nucleon-nucleon potential. The first reason is that at least the top-down models such as ours have very few adjustable parameters and should be very predictive and precise. This is not to say, of course, that the latter would be more accurate. On the contrary, such top-down holographic computations are less likely to be near the correct low energy QCD physics.

What we aim to build is not the most phenomenologically correct model, but rather those which can give us insights and results from top-down approach and that cannot be given by any of conventional phenomenological methods. The universal $1 / r^{2}$ repulsive core above is one fine such example. The conventional chiral perturbation, for instance, generates $1 / r^{3}$ type repulsive core from exchange rho mesons via tensor couplings as well as $1 / r$ from omega meson exchange [38], whose precise behavior, however, should be taken with a grain of salt at short distance below the mass of the nucleon. In practice, one further introduces other short-distance cut-offs to better simulate nature. Whether or not the holographic $1 / r^{2}$ repulsive core found by above holographic computations represents a better solution to this short-distance treatment of nuclear force and a more faithful image of real QCD is a matter to be settled eventually by lattice QCD simulations or by experiments.

\section{Conclusion}

We have derived a $\mathrm{U}(k)$ matrix model (2.1) which describes $k$-baryon systems at short distance, by considering open string theory on the wrapped baryon vertex D-branes embedded in the D4-D8 model of large $N_{c}$ holographic QCD. With this matrix model, we computed the holographic size and the spectrum of the baryon $(k=1)$, and also short-distance nuclear force $(k=2)$. The latter exhibits a repulsive core, which has been quite important in nuclear and hadron physics for long years.

This model complements the Yang-Mills soliton picture of the holographic baryon [1719], in that it is capable of addressing short-distance behavior such as the crucial hadronic size estimate of the baryon. Recall that the size found in these studies turned out to be comparable to the (appropriately warped) string scale, which is too small to be justified by the Yang-Mills method used there. Our new matrix model is trustworthy well below the string scale, in the opposite end of length scale, and represents an opportunity to check whether the soliton picture gave us the correct estimates or not. As we summarize shortly, the matrix model estimate gave us essentially the same size up to a numerical factor of $(5 / 4)^{1 / 4} \sim 1$, showing that stringy correction does very little to correct the soliton picture. This is very fortunate for the underlying D4-D8 holographic QCD since, at least for small number of baryons, the soliton picture has generated many predictions that agreed with nuclear data [19-21, 23]. This is by far the best evidence we know of that justifies study 
of baryons in holographic QCD, despite the latter's huge mass and small size in the large $N_{c}$ and large $\lambda$ limit.

A distinguished character of our matrix model is its simplicity. In particular, the form of the matrix model does not depend on the number of the baryons described simply changing the rank of the matrix allows one to treat larger number of baryons. By generalizing the method we did in this paper, we can handle general $k$-body systems. This is significant simplicity compared with the soliton picture, where handling general $k$-solitons are quite painful due to the significant increase of the number of moduli parameters.

Therefore it is natural to apply our matrix model analysis to more general $k$-body systems. In nuclear physics, the role of 3-body forces is very important. For example, for few-body nuclear bound states (light nuclei), the three-body forces are known to play a crucial role. Three-body forces in the soliton picture in the D4-D8 system were derived in [39]. However, in that paper the isospins and spins were treated classically due to the complications induced by the increase of the number of moduli parameters, so the analysis was not complete. Applying our matrix model, it is possible to treat generic spins and isospins quantum-mechanically [40]. It would be interesting to see how the quantum spin and isospin effects modify the previous soliton picture results [39].

It would also be interesting to consider a large $k$ limit, where the states are similar to extremely heavy nuclei or a core of neutron stars. The reader may wonder the effect of a back-reaction in that limit. Taking the large $k$ limit certainly cause the back-reaction of the multiple D4'-branes in D8-branes in D4-D8 system. However as we have seen, in our system, we have no supersymmetric leftover, and as a result, there is a repulsive forces between D4'-branes. So the D4'-branes cannot approach closer than $\rho=\mathcal{O}(1 / \sqrt{\lambda}) \sim l_{s}^{\text {eff }}$, which is too large compared with $\rho \sim\left(l_{s}^{\text {eff }}\right)^{2}$ for Maldacena's decoupling limit [8] in the $l_{s}^{\text {eff }} \rightarrow 0$ limit. ${ }^{16}$ Therefore the back-reaction is expected not so strong enough to make a back-reacted gravitational throat.

Going back to the results for $k=1,2$ in this work, we note that the results agree with the previous soliton approach qualitatively, and in some case quantitatively also. The central isospin-independent part of the repulsive core is one example. The holographic size squared of the baryon also turned out to be larger only by a fraction which, considering the vast separation of scales between the two approaches by a factor of $\lambda^{1 / 2}$, seems pretty innocuous. This tells us that physics of baryon in D4-D8 holographic QCD is relatively stable against corrections. This stability of the baryon physics against short distance corrections was previously anticipated and argued for on the basis of an approximate supersymmetry [26], and is borne out in our new matrix model approach. Without such stability, study of baryons in holographic QCD would have been very difficult and cumbersome.

On the other hand, the new results also show nontrivial change of key observables, which cannot be ignored. From the viewpoint of low energy effective theory in terms of mesons and baryons, the change can be summarized in the large $N_{c}$ limit as a universal and multiplicative increase by $(5 / 4)^{1 / 2}$ of certain meson-nucleon-nucleon couplings. These are

\footnotetext{
${ }^{16}$ This argument could break down for finite $l_{s}^{\text {eff }}$ or in supersymmetric cases and we may consider a holographic dual of the multi-baryon system in the $k \rightarrow \infty$ limit. It leads to "holographic nuclei" [6, 7].
} 
couplings denoted as $g^{(I I)}$ in section 3.5, and include the leading axial couplings to pions, all isotriplet axial vector couplings of the operator dimension four, and the dimension-five derivative couplings to isotriplet vector mesons. In particular, the change came about not as subleading $1 / N_{c}$ corrections, but a change in coefficients of the leading terms.

The reader may wonder whether the matrix model can describe only the short distance physics, such as the repulsive core of the nucleon. As we argued in section 3.5 and reiterated above, the model is capable of addressing the long-distance physics of soft-meson processes, via the single quantity $\rho^{2}$, so in that indirect sense also tells us much about baryons far away from one another. An entirely different question is whether we can use the matrix model directly to compute interaction, as we did in section 4, between well-separated baryons without resorting to intermediate mesons. This is particularly important when we wish to consider large $k$ physics where meson exchange viewpoint would become quickly intractable.

The above matrix model does take into account the dual warped geometry of the pure QCD background, so it already knows about effect of glues at energy scale $M_{\mathrm{KK}}$. Yet, how to emulate the long-distance physics due to exchange of pions and other light mesons directly by manipulating the current matrix model is a more challenging problem. For example, in a similar problem of D0-brane interactions, it was the one-loop effect in the open string side that emulated the long distance graviton exchange between them. This agreement was explained by the combination of supersymmetry and the worldsheet channel duality [33]. In the present case, we must emulate an exchange of D8-D8 open strings by a matrix model of D4'-D4' and D4'-D8 open strings. This appears to be a qualitative different problem from the case of D0 interactions. Absence of unbroken supersymmetry probably make matters a bit worse also.

Nevertheless, we believe that this can be achieved in the matrix model with judicious insertions of effective operators and by considering quantum effects. Once this is done, on the other hand, our matrix model may prove to be much more versatile than the previous instanton soliton picture of baryons, since it can be more easily generalized to many nucleon systems. Nuclei with generic atomic number $k$ and perhaps other dense matter systems would be more accessible that way. Perhaps we can eventually construct a $\mathrm{U}(k)$ matrix model, where attractive long-distance forces and repulsive short-distance forces are carefully balanced and produces,say, helium nucleus and carbon nucleus.

In this context, we finally comment that we only made use of bosonic fields in this work, even though there should be fermionic partners in D4'-D8 matrix theory. For this work, we did not need loop computation, which justifies this truncation. For a more complete matrix model capable of directly dealing with baryons at large separation, we probably need to consider fermionic fields as well, which is beyond the scope of this paper.

Eventually we hope to be able to compute other interesting quantities in our matrix model, such as currents associated with chiral symmetry, energy-momentum tensor and equations of states from that. In particular, equations of states with large baryon number $k$ are important to understand the dynamics of astrophysical compact starts, such as neutron stars. 


\section{Acknowledgments}

We would like to thank Yukawa Institute in Kyoto university and the organizers of the workshop on Branes, Strings and Black Holes, where this project started. K.H. is grateful to D. Tong for a discussion, and would like to thank DAMTP in university of Cambridge, National Taiwan Normal university, IPMU in university of Tokyo and CERN for hospitality. N.I. thanks RIKEN for hospitality. P.Y. was supported in part by the National Research Foundation of Korea (NRF) funded by the Ministry of Education, Science and Technology via the Center for Quantum Spacetime (grant number 2005-0049409), Basic Science Research Program (grant number 2010-0013526), and Basic Research Promotion Fund (grant number KRF-2007-314-C00052) .

Open Access. This article is distributed under the terms of the Creative Commons Attribution Noncommercial License which permits any noncommercial use, distribution, and reproduction in any medium, provided the original author(s) and source are credited.

\section{References}

[1] G. 't Hooft, A Planar Diagram Theory For Strong Interactions, Nucl. Phys. B 72 (1974) 461 [SPIRES].

[2] E. Witten, Baryons in the 1/N Expansion, Nucl. Phys. B 160 (1979) 57 [SPIRES].

[3] E. Witten, Baryons and branes in anti de Sitter space, JHEP 07 (1998) 006 [hep-th/9805112] [SPIRES].

[4] D.J. Gross and H. Ooguri, Aspects of large- $N$ gauge theory dynamics as seen by string theory, Phys. Rev. D 58 (1998) 106002 [hep-th/9805129] [SPIRES].

[5] M.F. Atiyah, N.J. Hitchin, V.G. Drinfeld and Y.I. Manin, Construction of instantons, Phys. Lett. A 65 (1978) 185 [SPIRES].

[6] K. Hashimoto, Holographic Nuclei, Prog. Theor. Phys. 121 (2009) 241 [arXiv:0809.3141] [SPIRES].

[7] K. Hashimoto, Holographic Nuclei : Supersymmetric Examples, JHEP 12 (2009) 065 [arXiv: 0910.2303] [SPIRES].

[8] J.M. Maldacena, The large-N limit of superconformal field theories and supergravity, Adv. Theor. Math. Phys. 2 (1998) 231 [Int. J. Theor. Phys. 38 (1999) 1113] [hep-th/9711200] [SPIRES].

[9] S.S. Gubser, I.R. Klebanov and A.M. Polyakov, Gauge theory correlators from non-critical string theory, Phys. Lett. B 428 (1998) 105 [hep-th/9802109] [SPIRES].

[10] E. Witten, Anti-de Sitter space and holography, Adv. Theor. Math. Phys. 2 (1998) 253 [hep-th/9802150] [SPIRES].

[11] T. Sakai and S. Sugimoto, Low energy hadron physics in holographic QCD, Prog. Theor. Phys. 113 (2005) 843 [hep-th/0412141] [SPIRES].

[12] E. Witten, Anti-de Sitter space, thermal phase transition and confinement in gauge theories, Adv. Theor. Math. Phys. 2 (1998) 505 [hep-th/9803131] [SPIRES]. 
[13] T.H.R. Skyrme, A Unified Field Theory Of Mesons And Baryons, Nucl. Phys. 31 (1962) 556 [SPIRES].

[14] T.H.R. Skyrme, A Nonlinear field theory, Proc. Roy. Soc. Lond. A 260 (1961) 127 [SPIRES].

[15] T.H.R. Skyrme, Particle states of a quantized meson field, Proc. Roy. Soc. Lond. A 262 (1961) 237 [SPIRES].

[16] T. Sakai and S. Sugimoto, More on a holographic dual of QCD, Prog. Theor. Phys. 114 (2005) 1083 [hep-th/0507073] [SPIRES].

[17] D.K. Hong, M. Rho, H.-U. Yee and P. Yi, Chiral dynamics of baryons from string theory, Phys. Rev. D 76 (2007) 061901 [hep-th/0701276] [SPIRES].

[18] H. Hata, T. Sakai, S. Sugimoto and S. Yamato, Baryons from instantons in holographic QCD, Prog. Theor. Phys. 117 (2007) 1157 [hep-th/0701280] [SPIRES].

[19] D.K. Hong, M. Rho, H.-U. Yee and P. Yi, Dynamics of Baryons from String Theory and Vector Dominance, JHEP 09 (2007) 063 [arXiv:0705.2632] [SPIRES].

[20] D.K. Hong, M. Rho, H.-U. Yee and P. Yi, Nucleon Form Factors and Hidden Symmetry in Holographic QCD, Phys. Rev. D 77 (2008) 014030 [arXiv:0710.4615] [SPIRES].

[21] K. Hashimoto, T. Sakai and S. Sugimoto, Holographic Baryons : Static Properties and Form Factors from Gauge/String Duality, Prog. Theor. Phys. 120 (2008) 1093 [arXiv:0806.3122] [SPIRES].

[22] K. Hashimoto, T. Sakai and S. Sugimoto, Nuclear Force from String Theory, Prog. Theor. Phys. 122 (2009) 427 [arXiv:0901.4449] [SPIRES].

[23] Y. Kim, S. Lee and P. Yi, Holographic Deuteron and Nucleon-Nucleon Potential, JHEP 04 (2009) 086 [arXiv: 0902 .4048] [SPIRES].

[24] J. Park and P. Yi, A Holographic QCD and Excited Baryons from String Theory, JHEP 06 (2008) 011 [arXiv:0804.2926] [SPIRES].

[25] H.R. Grigoryan, T.S.H. Lee and H.-U. Yee, Electromagnetic Nucleon-to-Delta Transition in Holographic QCD, Phys. Rev. D 80 (2009) 055006 [arXiv: 0904.3710] [SPIRES].

[26] P. Yi, Holographic Baryons, arXiv:0902.4515 [SPIRES].

[27] E. Witten, Small Instantons in String Theory, Nucl. Phys. B 460 (1996) 541 [hep-th/9511030] [SPIRES].

[28] M.R. Douglas, Branes within branes, hep-th/9512077 [SPIRES].

[29] B. Collie and D. Tong, Instantons, Fermions and Chern-Simons Terms, JHEP 07 (2008) 015 [arXiv:0804.1772] [SPIRES].

[30] S. Kim, K.M. Lee and S. Lee, Dyonic Instantons in 5-dim Yang-Mills Chern-Simons Theories, JHEP 08 (2008) 064 [arXiv: 0804.1207] [SPIRES].

[31] G.W. Gibbons and K.-i. Maeda, Black Holes and Membranes in Higher Dimensional Theories with Dilaton Fields, Nucl. Phys. B 298 (1988) 741 [SPIRES].

[32] N. Dorey, T.J. Hollowood, V.V. Khoze and M.P. Mattis, The calculus of many instantons, Phys. Rept. 371 (2002) 231 [hep-th/0206063] [SPIRES].

[33] T. Banks, W. Fischler, S.H. Shenker and L. Susskind, $M$ theory as a matrix model: A conjecture, Phys. Rev. D 55 (1997) 5112 [hep-th/9610043] [SPIRES]. 
[34] N. Ishibashi, H. Kawai, Y. Kitazawa and A. Tsuchiya, A large- $N$ reduced model as superstring, Nucl. Phys. B 498 (1997) 467 [hep-th/9612115] [SPIRES].

[35] K. Hashimoto and N. Iizuka, Nucleon Statistics in Holographic QCD : Aharonov-Bohm Effect in a Matrix Model, arXiv:1006.3612 [SPIRES].

[36] K.-Y. Kim and I. Zahed, Nucleon-Nucleon Potential from Holography, JHEP 03 (2009) 131 [arXiv: 0901.0012] [SPIRES].

[37] Y. Kim, S. Lee and P. Yi, Nucleon-nucleon potential in holographic QCD, Nucl. Phys. A $\mathbf{8 4 4}$ (2010) 224C [SPIRES].

[38] T. Ericsson and W. Weise, Pions and Nuclei, Clarendon Press, Oxford U.K. (1988).

[39] K. Hashimoto, N. Iizuka and T. Nakatsukasa, N-Body Nuclear Forces at Short Distances in Holographic QCD, Phys. Rev. D 81 (2010) 106003 [arXiv:0911.1035] [SPIRES].

[40] K. Hashimoto and N. Iizuka, Three-Body Nuclear Forces from a Matrix Model, arXiv: 1005. 4412 [SPIRES]. 